\title{
Interactive Inverse Design Optimization of Fuselage Shape for Low-Boom Supersonic Concepts
}

\author{
Wu Li, ${ }^{*}$ Elwood Shields, ${ }^{\dagger}$ and Daniel Le ${ }^{\ddagger}$
}

\begin{abstract}
There are several research papers on how to design a supersonic configuration that has desirable low-boom characteristics as determined by the Seebass-George-Darden boom minimization theory (SGD theory). The low-boom signatures predicted by the SGD theory could be realized by wing-fuselage configurations. However, for a given low-boom signature generated by the SGD theory, it is still an open question whether one could develop a feasible aircraft configuration with nacelles and tails that has a similar low-boom ground signature as that predicted by the SGD theory.

Past attempts indicated that no feasible aircraft configuration with nacelles and tails would have a total equivalent area distribution matching one of the equivalent area distributions corresponding to the low-boom ground signatures determined by the SGD theory. There are essentially three alternative methods for generating a supersonic concept with a shaped boom ground signature: (i) use a direct optimization method that minimizes numerical figures of merit for low-boom characteristics, (ii) construct new "realizable" target equivalent area distributions (or near-field pressure distributions) that result in shaped boom ground signatures, and (iii) develop new tools to help designers find acceptable low-boom configurations. There were many attempts, with mixed results, using the first two methods to obtain supersonic configurations that have shaped boom ground signatures.

This paper introduces a tool called BOSS (Boom Optimization using Smoothest Shape modifications). BOSS utilizes interactive inverse design optimization to develop a fuselage shape that yields a low-boom aircraft configuration. The paper also demonstrates how BOSS could be used to help design realistic aircraft concepts with low-boom ground signatures. A fundamental reason for developing BOSS is the need to generate feasible low-boom conceptual designs that are appropriate for further refinement using CFD-based preliminary design methods. BOSS was not developed to provide a numerical solution to the inverse design problem. Instead, BOSS was intended to help designers find the "right" configuration among infinitely many possible configurations that are equally good using any numerical figure of merit.
\end{abstract}

BOSS uses the smoothest shape modification strategy for modifying the fuselage radius distribution at 100 or more longitudinal locations to find a smooth fuselage shape that reduces the discrepancies between the design and target equivalent area distributions over any specified range of effective distance. For any given supersonic concept (with wing, fuselage, nacelles, tails, and/or canards), a designer can examine the differences between the design and target equivalent areas, decide which part of the design equivalent area curve needs to be modified, choose a desirable rate for the reduction of the discrepancies over the specified range, and select a parameter for smoothness control of the fuselage shape. BOSS will then generate a fuselage shape based on the designer's inputs in a matter of seconds. If the generated solution is not acceptable, the designer can work on a different part of the equivalent area curve, change the rate of reduction, or relax the smoothness control until a desirable solution is found. The new configuration will be analyzed by PBOOM (a sonic boom analysis code) to see whether it has an acceptable low-boom ground signature. If not, the designer can use BOSS to further reduce the differences between the design and target equivalent areas until the configuration has an acceptable low-boom ground signature. Using BOSS and PBOOM, the designer can generate a realistic, smooth fuselage shape that results in a supersonic configuration with a low-boom ground signature in a few hours. In addition, a designer can use BOSS to quickly eliminate any configuration that cannot achieve low-boom characteristics with fuselage shaping alone.

For any given wing planform and layout of aircraft components, BOSS reduces the design time of low-boom supersonic concepts from months to hours. More importantly, BOSS allows a quick closure of the fuselage shaping process because BOSS will let the designer see how much deterioration of the fuselage shape is necessary for any further reduction of the discrepancies between the design and target equivalent area distributions.

A conceptual design case study is documented to demonstrate how BOSS can be used to develop a low-boom supersonic concept from a low-drag supersonic concept. The paper also contains a study on how perturbations in the equivalent area distribution affect the ground signature shape and how new target area distributions for low-boom signatures can be constructed using superposition of equivalent area distributions determined by the SGD theory.

\footnotetext{
*The corresponding author, email: w.li@nasa.gov

NASA Langley Research Center, Hampton, Virginia, USA

$\dagger$ Swales Aerospace, Hampton, Virginia, USA

$¥$ University of Virginia, Charlottesville, Virginia, USA
} 


\title{
Interactive Inverse Design Optimization of Fuselage Shape for Low-Boom Supersonic Concepts
}

\author{
Wu Li, ${ }^{*}$ Elwood Shields ${ }^{\dagger}$ and Daniel Le ${ }^{\ddagger}$
}

\begin{abstract}
This paper introduces a tool called BOSS (Boom Optimization using Smoothest Shape modifications). BOSS utilizes interactive inverse design optimization to develop a fuselage shape that yields a low-boom aircraft configuration. A fundamental reason for developing BOSS is the need to generate feasible low-boom conceptual designs that are appropriate for further refinement using computational fluid dynamics (CFD) based preliminary design methods. BOSS was not developed to provide a numerical solution to the inverse design problem. Instead, BOSS was intended to help designers find the "right" configuration among an infinite number of possible configurations that are equally good using any numerical figure of merit. BOSS uses the smoothest shape modification strategy for modifying the fuselage radius distribution at 100 or more longitudinal locations to find a smooth fuselage shape that reduces the discrepancies between the design and target equivalent area distributions over any specified range of effective distance. For any given supersonic concept (with wing, fuselage, nacelles, tails, and/or canards), a designer can examine the differences between the design and target equivalent areas, decide which part of the design equivalent area curve needs to be modified, choose a desirable rate for the reduction of the discrepancies over the specified range, and select a parameter for smoothness control of the fuselage shape. BOSS will then generate a fuselage shape based on the designer's inputs in a matter of seconds. Using BOSS, within a few hours, a designer can either generate a realistic fuselage shape that yields a supersonic configuration with a low-boom ground signature or quickly eliminate any configuration that cannot achieve low-boom characteristics with fuselage shaping alone. A conceptual design case study is documented to demonstrate how BOSS can be used to develop a low-boom supersonic concept from a low-drag supersonic concept. The paper also contains a study on how perturbations in the equivalent area distribution affect the ground signature shape and how new target area distributions for low-boom signatures can be constructed using superposition of equivalent area distributions derived from the Seebass-George-Darden (SGD) theory.
\end{abstract}

\section{Nomenclature}

A normal area

$A_{e} \quad$ equivalent area

$G(\mathbf{r}) \quad$ least squares error of mismatch of equivalent areas for given $\mathbf{r}$

$\frac{\partial G}{\partial \mathbf{r}}\left(\mathbf{r}_{k}\right) \quad$ gradient vector of $G(\mathbf{r})$ with respect to $\mathbf{r}$ evaluated at $\mathbf{r}_{k}$

$L(\mathbf{r}) \quad$ linear term in Taylor expansion of $G(\mathbf{r})$

$f(x) \quad$ camber location of fuselage at $x$

$l \quad$ total length of fuselage

$l_{e} \quad$ effective length of aircraft

$\mathbf{r} \quad$ vector of radii at a finite number of $x$ locations

*The corresponding author, email: w.li@nasa.gov

NASA Langley Research Center, Hampton, Virginia, USA

† Swales Aerospace, Hampton, Virginia, USA

$\ddagger$ University of Virginia, Charlottesville, Virginia, USA 


$\begin{array}{ll}r(x) & \text { radius of a circular cross-section of fuselage at } x \\ x_{e} & \text { effective distance along the longitudinal direction } \\ s & \text { smoothness control parameter for fuselage shape modifications } \\ x, y, z & \text { coordinates of a point in space } \\ \Delta A & \text { change in area } \\ \Delta r(x) & \text { change in radius at } x \\ \Delta r^{\prime \prime}(x) & \text { the second derivative of } \Delta r(x) \\ \Delta r^{(3)}(x) & \text { third derivative of } \Delta r(x) \\ \delta & \text { positive constant bound for } \Delta r^{(3)}(x) \\ \mathcal{E} & \text { envelope region generated by low-boom equivalent area distributions } \\ \lambda_{s} & \text { positive multiple of bound for } \Delta r^{(3)}(x) \\ \rho & \text { reduction rate of } G(\mathbf{r}) \text { in BOSS } \\ \langle\mathbf{a}, \mathbf{b}\rangle & \text { dot product of two vectors a and } \mathbf{b}\end{array}$

\section{Subscripts and Superscripts}

$i \quad$ index for $i$ th component of vector

$k \quad$ index for an instance of vector $\mathbf{r}$

$f \quad$ superscript for fuselage shape

\section{Introduction}

Many research papers address how to design a supersonic configuration that has desirable low-boom characteristics as determined by the Seebass-George-Darden (SGD) boom minimization theory. ${ }^{1-3}$ The lowboom signatures predicted by the SGD theory could be realized by wing-fuselage configurations. However, for a given low-boom signature generated by the SGD theory, the question still exists for whether one could develop a feasible aircraft configuration with nacelles and tails that has a low-boom ground signature similar to that predicted by the SGD theory. In this paper, Whitham's boom propagation method ${ }^{4,5}$ is used for boom signature analysis.

Past attempts have demonstrated little success in creating feasible aircraft configurations with nacelles and tails that have a total equivalent area distribution matching one of the target equivalent area distributions derived from the SGD theory. Essentially three design methods exist for generating a supersonic concept with a shaped boom ground signature: (i) use a direct optimization method that minimizes numerical figures of merit for low-boom characteristics, (ii) construct new, "realizable" target equivalent area distributions (or target near-field pressure distributions) that result in shaped boom ground signatures, and (iii) develop new tools to help designers find acceptable low-boom configurations. Many unsuccessful attempts have been made using the first two methods to obtain supersonic configurations that have shaped boom ground signatures. In this paper, we introduce a tool, called BOSS (Boom Optimization using Smoothest Shape modifications), for interactive inverse design optimization of fuselage shape for developing low-boom concepts and demonstrate how it could help designers develop realistic aircraft concepts with low-boom ground signatures.

McMasters and Cummings point out the need for developing optimization strategies "that may be unique to the aircraft industry, which take advantage of the assumptions and techniques that airplane designers use, rather than letting a computer churn away and come up with theoretically possible, but practically impossible, configurations." (See page 14 of ref. 6.) BOSS is not developed to provide a numerical solution to the inverse design problem. Instead, BOSS tries to help designers find the "right" configuration among an infinite number of possible configurations that are essentially equal using any numerical figure of merit.

This paper is organized as follows. Sections II-IV provide a brief survey of various numerical methods for design of low-boom supersonic concepts: inverse design by matching equivalent area distributions, inverse design by matching near-field pressure distributions, and numerical optimization for sonic-boom minimization. 
The interactive inverse design process for low-boom supersonic concepts using BOSS is discussed in Section V. To demonstrate how BOSS can assist conceptual designers in generating low-boom concepts, Section VI includes a case study that demonstrates the application of BOSS in developing a low-boom supersonic concept from a low-drag supersonic concept. In Section VII, we attempt to understand why similarly shaped boom signatures can have different $A_{e}$ distributions and why "saw-tooth" oscillations in boom signature are unavoidable. Concluding remarks are given in Section VIII.

\section{Inverse Design by Matching Equivalent Area Distributions}

The SGD boom minimization theory ${ }^{1-3}$ can be used to generate equivalent area distributions that minimize sonic boom on the ground when analyzed using Whitham's boom propagation method. ${ }^{4,5}$ Darden's main contributions are the extension of Seebass and George's theory from a uniform and isothermal atmosphere to a real atmosphere ${ }^{2}$ and a more flexible form of Seebass and George's F-function that allows a low-boom configuration with a sharper nose rather than the blunt nose required by Seebass and George's theory. ${ }^{3}$ In figure 8 of ref. 3, for a body of revolution, Darden showed that drag caused by nose bluntness can be reduced with a marginal increase of the sonic-boom pressure strength. Seebass and Argrow ${ }^{7}$ gave a comprehensive review of the historical development of the SGD theory and called it the classical JonesSeebass-George-Darden theory. Most studies on inverse design of low-boom concepts are based on Darden's area distribution for a ramp ground signature. (See refs. 8-14.)

Two empirical modifications of the sonic-boom-minimizing F-functions were made to incorporate some practical considerations in design of low-boom configurations. The first is a hybrid combination of flattop and ramp F-functions. For the same initial overpressure level, it was observed that ramp signatures lead to a shorter supersonic aircraft while flattop signatures are less sensitive to atmospheric changes in temperature. ${ }^{15,16}$ (See also page 3 of ref. 17.) To capture the benefits of both flattop and ramp signatures, Haglund developed ${ }^{15,16}$ a hybrid F-function for sonic-boom minimization. The ground signature for a hybrid F-function has a flat segment near the initial pressure peak, followed by a ramp. (See figure 1(a).) The second modification is to use a conical fuselage nose ${ }^{18}$ instead of the cusp-shaped fuselage nose used by Darden. These modifications provide additional flexibilities for conceptual design of low-boom configurations. (See ref. 18.) For example, the hybrid F-function allows designers to do tradeoffs between the initial overpressure and the maximum overpressure in the ground signature, as well as the shape of the target equivalent area distribution. Figure 1(a) shows ramp, hybrid, and flattop signatures using the following parameters in F-functions defined of ref. 18: effective length $\left(l_{e}\right)$ of $111 \mathrm{ft}$, the fuselage nose-cone length $\left(y_{f}\right)$ of $6 \mathrm{ft}$, ramp slope of 0.35 , initial cruise weight of 96,500 lbs, cruise altitude of 53,000 ft, and cruise Mach of 1.8. For this set of parameter values, if both the initial overpressure and the maximum overpressure are used as the figures of merit, then the ramp and flattop signatures become two end points of a Pareto frontier. (See figure 2.) The hybrid signatures fill the Pareto frontier if the length of the flat segment in the hybrid signature is varied. (See figure 1(a).)

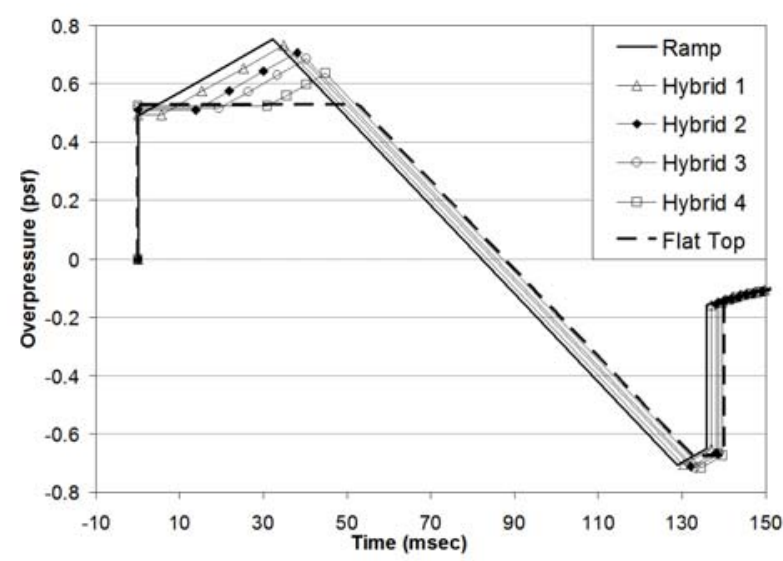

(a) Comparison of Signatures

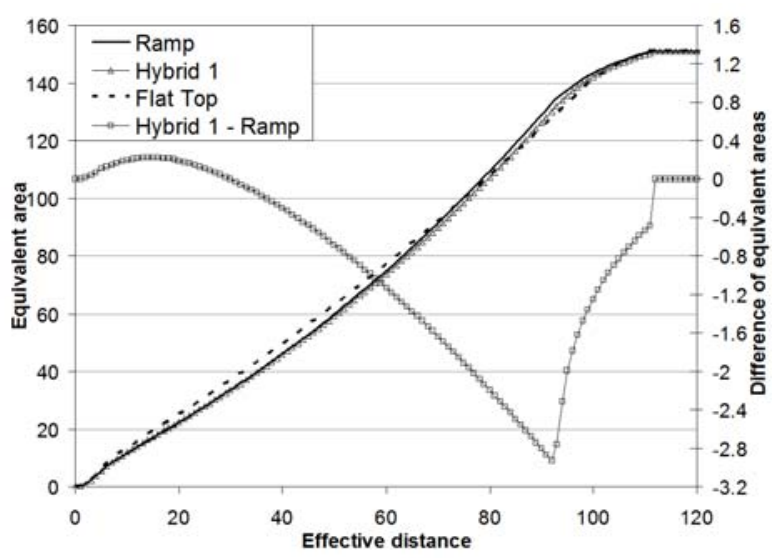

(b) Comparison of Equivalent Areas

Figure 1. Ramp, hybrid, and flattop ground signatures and the corresponding area distributions. 


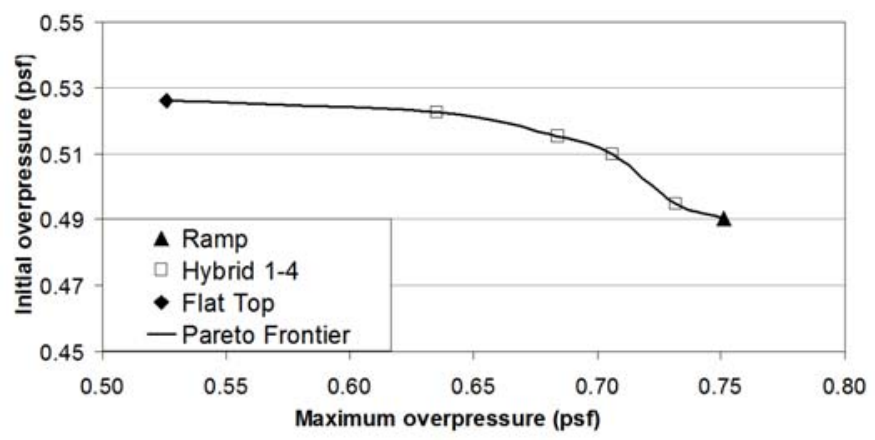

Figure 2. Pareto frontier for initial and maximum overpressures of flattop, ramp, and hybrid signatures.

The hybrid equivalent area in figure 1(b) corresponds to the hybrid point with an initial overpressure of $0.496 \mathrm{psf}$ in figure 2 or Hybrid 1 in figure 1(a) and is assumed to have the potential benefit of maintaining the level of the initial overpressure under atmospheric perturbations ${ }^{16}$ in comparison with the ramp signature. However, in reality, because the difference between the equivalent area distributions of the ramp and the hybrid signatures is quite small (see the secondary axis for the difference between the two area distributions in figure 1(b)), one might wonder whether such a difference would make any meaningful impact on the shape of the low-boom configuration. We will come back to this issue in the discussion of a low-boom configuration that is designed for the hybrid signature.

Shepard and Sullivan ${ }^{19}$ investigated the relationship between sonic-boom shapes and loudness. The main conclusion is that among all of the boom signatures with the same maximum overpressure and rise time, the subjective loudness levels of the flattop signature and the N-wave are the same, while the ramp and the hybrid signatures have lower loudness levels. Therefore, for trade-off studies of sonic-boom shapes during conceptual design, a comparison of loudness levels for various sonic-boom shapes that correspond to the Pareto points in figure 2 is more useful and provides insight into pros and cons of different sonic-boom shapes under the same set of design constraints. For all of the signatures in figure 1(a), the loudness levels are in the range of 85.3 to $85.9 \mathrm{PldB}$ computed using $A$-weighted frequency distributions.

Low-boom supersonic configurations that correspond to theoretical boom signatures could still be too long to be practical (e.g., see ref. 20). In an attempt to develop low-boom configurations with a reasonable length for supersonic business jets, Mack first proposed the idea of allowing a difference between the maximum equivalent area due to lift and the maximum equivalent area based on the boom minimization theory. ${ }^{21}$ This approach allows a shaped boom signature with a more practical configuration, at the expense of an increase in aft-body sonic-boom pressure strength when predicted by Whitham's F-function method. ${ }^{4,5}$ Mack discussed extensively the difficulties of designing a low-boom supersonic business jet. (See page 21 of ref. 21.) Significant differences were evident between the target hybrid ground signature and the predicted ground signature of the proposed low-boom supersonic business concept, even without nacelles. (See figure 13 of ref. 21.) A wind-tunnel model was constructed based on a watertight version of the conceptual design; the model was tested in the Langley Unitary Plan Wind Tunnel (see page 6 of ref. 22) and analyzed by Choi, Alonso, and Weide. ${ }^{22}$ The ground signatures of the wind-tunnel model, propagated from the measured near-field pressures by PCBoom3, are N-waves; the ground signatures of the wind-tunnel model, propagated from predicted near-field pressures, have a two-level staircase-type shape near the initial peak. (See figures $17-19$ of ref. 22.)

Numerical algorithms were proposed to modify the fuselage shape so that the total equivalent area distribution of the resultant configuration matches a target area distribution. Barger and Adams's methods ${ }^{23}$ were based on a fixed-point iteration procedure for changing the fuselage so that the equivalent area of the fuselage volume matches a target distribution. Barger and Adams developed two numerical algorithms. One algorithm is slow to converge and has difficulty matching the target area distribution but generates a smooth fuselage; the other one converges quickly but generates a wavy fuselage that more closely matches the target area distribution. As an example, they constructed two different fuselage shapes with circular cross sections that have the same target area distribution. (See figures 4 and 5 of ref. 23.) Their study has some negative implications on numerical algorithms for modifying fuselage shapes to match a target area distribution: the algorithm might numerically generate a fuselage shape that either doesn't match the target area distribution 
or is not realistic.

Rather than developing an algorithm for numerical fuselage solutions for equivalent area matching, Mack and Needleman ${ }^{24}$ used a semiempirical method for modifying the fuselage radius distribution to match the target equivalent area distribution. This method could have significant errors if the fuselage violates the slender-body assumption (i.e., a relatively flat camber line and small variations in fuselage radius distribution). This semiempirical method was used to generate various low-boom supersonic concepts, but additional experience-based area trimming might be required to obtain a desirable low-boom configuration. (See refs. 24 and 21.)

Recently, Rallabhandi and Mavris ${ }^{25}$ tried a new approach for low-boom supersonic conceptual design. A new flexible form of the F-function was used to generate new target total equivalent area distributions for sonic-boom minimization. The fuselage shape was then modified to meet the nose shape. After a suitable match was obtained, the fuselage shape was frozen and the other components were perturbed to match the target area distribution as closely as possible. However, this approach could not obtain a supersonic concept for which the total equivalent area was a desirable match to the target area distribution. (See figure 25 of ref. 25.) In the same paper ${ }^{25}$ a parallel genetic algorithm was used to find low-boom supersonic concepts, but no specific shape parameters were given.

\section{Inverse Design by Matching Near-Field Pressure Distributions}

The limitations of Whitham's F-function method for sonic-boom prediction are well-known. (See, for example, ref. 26.) A remedy is to use a three-dimensional flow solver to predict the near-field pressure distributions and then extrapolate the near-field pressure distributions to the ground using either Thomas's code $^{27}$ or PCBOOM. ${ }^{28}$

Inverse design processes for fuselage shaping of low-boom supersonic concepts, coupled with CFD analysis to match near-field pressure targets, have been studied for many years. (See refs. 10-12,14,20,29,30.)

Makino et al. ${ }^{10}$ studied a wing-body configuration based on Darden's target area distribution for a flattop ground signature with a blunt nose. (See the section on Aircraft Configuration of ref. 10.) Figure 8 of ref. 10 shows an almost perfect match of the configuration's equivalent area distribution and the target everywhere except near the end of the configuration. The predicted ground signature is generated by Thomas's code using a near-field pressure distribution at six body lengths below the configuration. Significant differences are seen between the predicted ground signature and the target flattop ground signature. (See figure 11 of ref. 10.) Specifically, the predicted maximum overpressure is $2.0 \mathrm{psf}$ instead of $1.0 \mathrm{psf}$ for the target signature. No information is provided on the ground signature predicted by Whitham's F-function method.

Later, Makino et al. ${ }^{29}$ used numerical optimization of fuselage geometry of the low-boom baseline discussed in ref. 10 to modify the sonic-boom signature predicted by Thomas's code using a near-field pressure distribution at six body lengths. They used numerical optimization to modify the fuselage shape over a selected segment of the fuselage. This process was repeated several times over different segments of the fuselage. The design variables of the optimization problem were eight control points of a B-spline representation of the shape of the fuselage segment that was being modified. (See page 672 of ref. 29 for details.) This effort is the first to show that a combination of heuristics (e.g., segment selection and choice of design variables) and numerical optimization could be useful for inverse design of low-boom configurations by matching a target near-field pressure distribution.

Nadarajah, Jameson, and Alonso ${ }^{31}$ used an adjoint method to shape a wing-body configuration to match a target near-field pressure distribution. They were able to reduce the sonic-boom level with a significant drag penalty. They then tried a composition of the drag coefficient and the pressure difference as the objective function for shape optimization using the adjoint method and showed that the method could reduce the peak pressures while maintaining the drag coefficient at the same level. However, the adjoint method could not generate a configuration with shaped boom signatures such as ramp or flattop signatures.

The only flight validation of any shaped boom signature is the modified F-5E Shaped Sonic Boom Demonstrator aircraft, which was designed with an initial configuration obtained by matching equivalent area distributions, followed by an iterative inverse design process of matching a target near-field pressure distribution. Here is a description of the design process:

"Initially, linear analysis tools were used to design the geometry shape of the aircraft to achieve the designed off-body pressures (changes to the area-distribution). This geometry was then analyzed with high-order CFD to compute the resultant off-body pressure signature of the candidate 
geometry. This became an iterative process, of geometry modifications, CFD analysis of geometry to compute pressure signatures, and then modifications of the geometry again." 14

The final design is an innovative configuration with great knowledge and experience behind it. Flight tests validated that the modified $\mathrm{F}-5 \mathrm{E}$ had a shaped sonic-boom signature versus the $\mathrm{N}$-wave signature of the baseline F-5E. ${ }^{32}$ The tests demonstrated for the first time that specialized aircraft shaping techniques can be used to tailor the sonic boom ground signature. (See ref. 33 for the origin and history of the shaped sonicboom demonstrator project.) Note that the shaping techniques only produced a set of candidate geometry shapes, and the wind-tunnel model shape was determined by consensus of a working group. ${ }^{33}$

\section{Numerical Optimization for Sonic-Boom Minimization}

There have been many studies on how to use direct optimization methods for generating low-boom configurations. ${ }^{13,31,34-39}$ An open question is "What objective function could lead to an optimal solution having a shaped boom signature with a low level of loudness?" Various forms of objective functions, including the initial overpressure, perceived loudness, drag, and so on, were proposed. Although each method is able to generate a better configuration in terms of the chosen objective function, no evidence indicates that any optimized configuration has a shaped low-boom signature except the configurations generated by Makino and Kroo. ${ }^{39}$

Makino and $\mathrm{Kroo}^{39}$ used a signature mold line evaluation method to seek a configuration with a low-boom ground signature. The documented process uses 12 design variables and genetic algorithms for shaping a low-drag configuration into a configuration for which the ground signature is within the mold lines of the target signature and the overpressure is equal to the minimum absolute value. Makino and Kroo were able to generate three configurations that have flattop, ramp, and hybrid signatures, respectively. The method is not a traditional inverse design based on the target equivalent area; but it can be considered a novel inverse design method for matching the envelope of the ground signature or the target signature mold lines. While experience indicates that many variables are needed to define a large design space a priori that contains configurations with low-boom ground signatures, Makino and Kroo used surprisingly few design variables to generate configurations with different low-boom characteristics.

\section{Interactive Optimization for Matching Equivalent Area Distributions}

In open literature, few references exist that document what and how design variables are modified to generate a low-boom configuration for a target area or pressure distribution. (See, for example, refs. 14,20, $21,23,29$.) The most detailed description was given by Makino et al., ${ }^{29}$ who used 14 numerical optimization iterations to modify 8 control points of a B-spline representation of the fuselage geometry over the selected segments of the fuselage. Recently, Makino and $\mathrm{Kroo}^{39}$ used an Akima spline representation of the radius distribution of an axisymmetric fuselage with seven control points for sonic-boom minimization using genetic algorithms. Their work showed very promising results for shaped boom configurations with tails and nacelles. One significant contribution of this work is the demonstration that, with an appropriate combination of the objective function and the design space parameterization, one could use a numerical optimization method to generate configurations with shaped boom signatures. However, one practical difficulty of using Makino and Kroo's optimization method is the choice of design variables and their ranges. Makino and Kroo stated in Section II of ref. 39 that "the ranges of the design variables are specified so that they can represent both low-drag and low-boom configurations while they are kept as small as possible for fast design convergence." In practice, identifying a priori the parametric design space that will include low-boom configurations is nearly impossible.

Because of the difficulty of designing a reasonably well-blended wing-body supersonic configuration for high-fidelity CFD analysis, the current conceptual design framework at Langley Research Center is based on a modified linear solution of forces and moments acting on twisted and cambered lifting surfaces of arbitrary planform for aerodynamic analysis and classical sonic-boom minimization theory for low-boom configuration design.

For a complete supersonic configuration with tails and nacelles, considerable time and effort is generally required to develop a low-boom configuration with some kind of practical considerations such as mission requirements. After overcoming the initial challenges of designing an aircraft layout, such as wing planform 
and nacelle locations (see, for example, refs. 21 and 20), one might find that the resulting configuration cannot be modified to match the target equivalent area distribution due to lack of volume between the target distribution and the equivalent area distribution due to lift. Several iterations are required before one gets a supersonic configuration that could be reshaped as a low-boom configuration. With this semiempirical method, ${ }^{24}$ a considerable amount of time can be spent shaping the fuselage to achieve relatively good agreement between the configuration's area distribution and the target, such as shown in figure 9 of ref. 21. However, significant differences can still exist between the corresponding ground signatures. (See figure 13 of ref. 21.) A similar mismatch between the configuration's equivalent area distribution and the target were also reported in ref. 20 because of the nacelles.

In this paper, we will only focus on developing an interactive optimization method for solving the inverse design problem of modifying the fuselage geometry to match a given target equivalent area distribution. In principle, the method is also applicable for solving the inverse design problem of modifying the fuselage geometry for a given near-field pressure distribution. The goal is to develop a practical optimization procedure for fuselage shaping that designers could use to develop low-boom concepts quickly, with a turnaround time of days instead of months, without any prior knowledge of any appropriate parameterization of the design space.

\section{A. Sonic-Boom Analysis Framework}

For a given gross takeoff weight of 96,500 lb, cruise altitude of 53,000 ft, cruise Mach of 1.8, an initial overpressure (0.5 psf), and two parameters defining the ground signature shape (the length of the flattop and the slope of the ramp), the sonic-boom minimization code, Hybrid, ${ }^{16}$ can generate a target equivalent area distribution for a configuration with a conic fuselage nose ${ }^{18}$ and minimum effective length. When other parameters are fixed, as the length of flattop is increased, either the effective length increases or the initial overpressure increases. The boom signatures shown in figure 1(a) were generated by changing the length of the flattop while fixing the effective length.

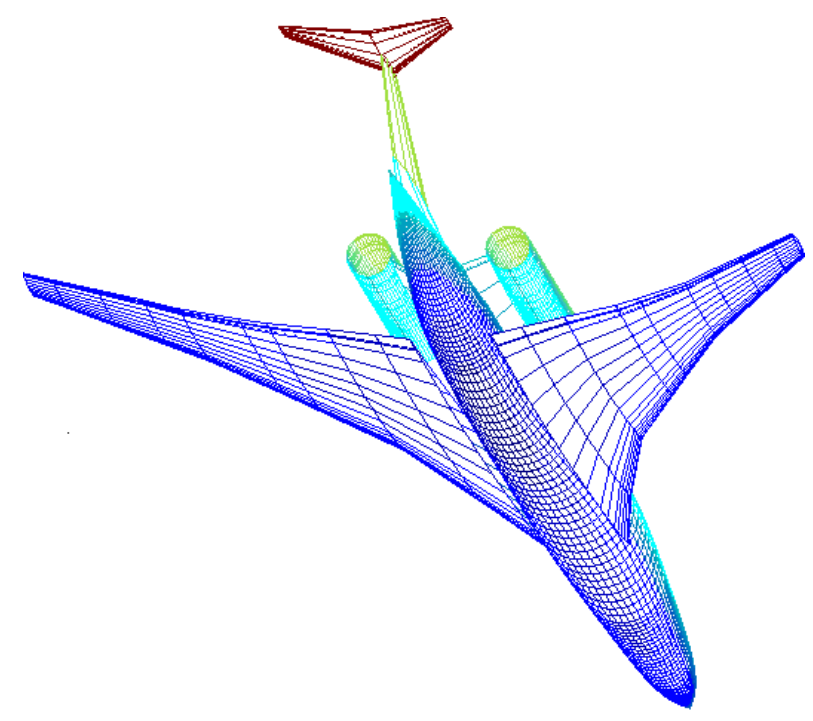

Figure 3. Aircraft geometry defined by grid points.

This particular aircraft geometry is defined by six aircraft components: fuselage, wing, nacelle (one circular shell and one pylon), and T-tail (one vertical tail and one horizontal tail), with no definition of intersections. Each component is defined by a fixed number of cross sections with a fixed number of points on each cross section. (See figure 3.) An analytical surface form of each component can be generated by a B-spline interpolation of the grid points, but we use the piecewise linear interpolation for surface points between the given grid points because it is sufficient for numerical calculation of the equivalent areas. If enough grid points are used, then the difference between a B-spline surface representation and the piecewise linear interpolation is negligible. 
In this paper, we only consider fuselages with circular cross sections. A circular fuselage is defined by a camber line $z=f(x)$ and a radius distribution $z=r(x)$. The fuselage surface points $(x, y, z)$ satisfy the equation $y^{2}+(z-f(x))^{2}=r(x)^{2}$. Of course, the graphs of $z=f(x)$ and $z=r(x)$ are piecewise linear interpolants of the given data sets $\left\{\left(x_{0}, f\left(x_{0}\right)\right),\left(x_{1}, f\left(x_{1}\right)\right), \ldots,\left(x_{n}, f\left(x_{n}\right)\right)\right\}$ and $\left\{\left(x_{0}, r_{0}\right),\left(x_{1}, r_{1}\right), \ldots,\left(x_{n}, r_{n}\right)\right\}$, respectively. Figure 4 shows a side view of the fuselage geometry. Note that the top and bottom surfaces are defined by $z_{u}=f(x)+r(x)$ and $z_{l}=f(x)-r(x)$, respectively. The camber line is designed to achieve three purposes: (i) at the cruise angle of attack, the front part of the fuselage behaves essentially like a body of revolution along the stream line; (ii) the cabin floor angle will be within some acceptable limits; and (iii) the aft part of the fuselage camber increases the effective length of the configuration and enhances ground clearance during rotation for takeoff and landing. These are knowledge-based practical considerations for a credible aircraft configuration.

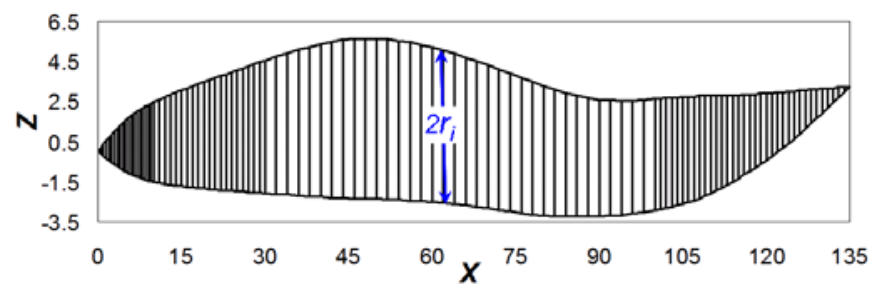

Figure 4. Side view of fuselage geometry defined by circular cross sections.

AWAVE $^{40}$ is used to evaluate the equivalent area distribution due to volume and a modified linear method $^{41}$ is used to evaluate the longitudinal lift distribution. These distributions are then used by $\mathrm{PBOOM}^{42}$ to evaluate the sonic-boom ground signature of the configuration.

A ModelCenter ${ }^{43}$ wrapper of the sonic-boom analysis was implemented by Lori Ozoroski and Karl Geiselhart of Aeronautics Systems Analysis Branch at NASA Langley Research Center. This wrapper is used as the basis for developing the current interactive inverse design optimization process for low-boom concepts.

The current low-boom conceptual design process first determines a layout for all of the components, designs a wing that sufficiently satisfies mission requirements, and then reshapes the fuselage (by modifying the discrete radius distribution $r_{i}$, as shown in figure 4) to achieve a low-boom configuration. Figure 5 shows the ModelCenter layout of the analysis and optimization tools used in the low-boom conceptual design process.

\section{B. Intrinsic Difficulties of Matching Equivalent Areas}

We shall use two numerical examples to illustrate the difficulties of finding an axisymmetric fuselage for which the equivalent area distribution matches a given target distribution. The first example shows a smooth target equivalent area distribution that cannot be matched by the equivalent area of any nonoscillatory fuselage shape; the second example shows that one smooth fuselage and another oscillatory fuselage have the same smooth equivalent area distribution. In this subsection, the equivalent area is computed under the assumptions that the cruise $M=1.8$ and the fuselage is at an angle of attack of 1.94 degrees. The fuselage geometry is defined by its radius distribution at 120 equally spaced longitudinal locations.

The radius distribution of an axisymmetric fuselage is defined as follows: $r(0)=r(135)=0, r(45)=$ $3, r(90)=2.7 ; r(x)$ is a quadratic function for $0 \leq x \leq 45, r(x)$ is a linear function for $45 \leq x \leq 90 ; r(x)$ is a quadratic function for $90 \leq x \leq 135$; and $r(x)$ has continuous first derivatives at $x=45$ and $x=90$. This radius distribution is plotted as "Original" in figure 6(a). Then a target equivalent area distribution is generated as follows: $A_{e \text {,target }}(x)=A_{e}(x)+\Delta A(x)$, where $A_{e}(x)$ is the equivalent area distribution of the original fuselage and $\Delta A(x)=0.0384(x-35)^{2}(x-40)^{2}$ if $35 \leq x \leq 40$ and $\Delta A(x)=0$ otherwise. An optimal fuselage is obtained by using a numerical optimization code that modifies the original fuselage for matching the equivalent area distributions.

Figure 6(b) shows the equivalent area distributions for the original and optimal fuselages, as well as the target distribution. Note that the distribution for the optimal fuselage closely matches the target distribution, with maximum error equaling about 0.3. (See the secondary axis in figure 6(b) for the area difference.) This value is about one percent for the relative fitting error. However, the optimal fuselage is quite oscillatory. 


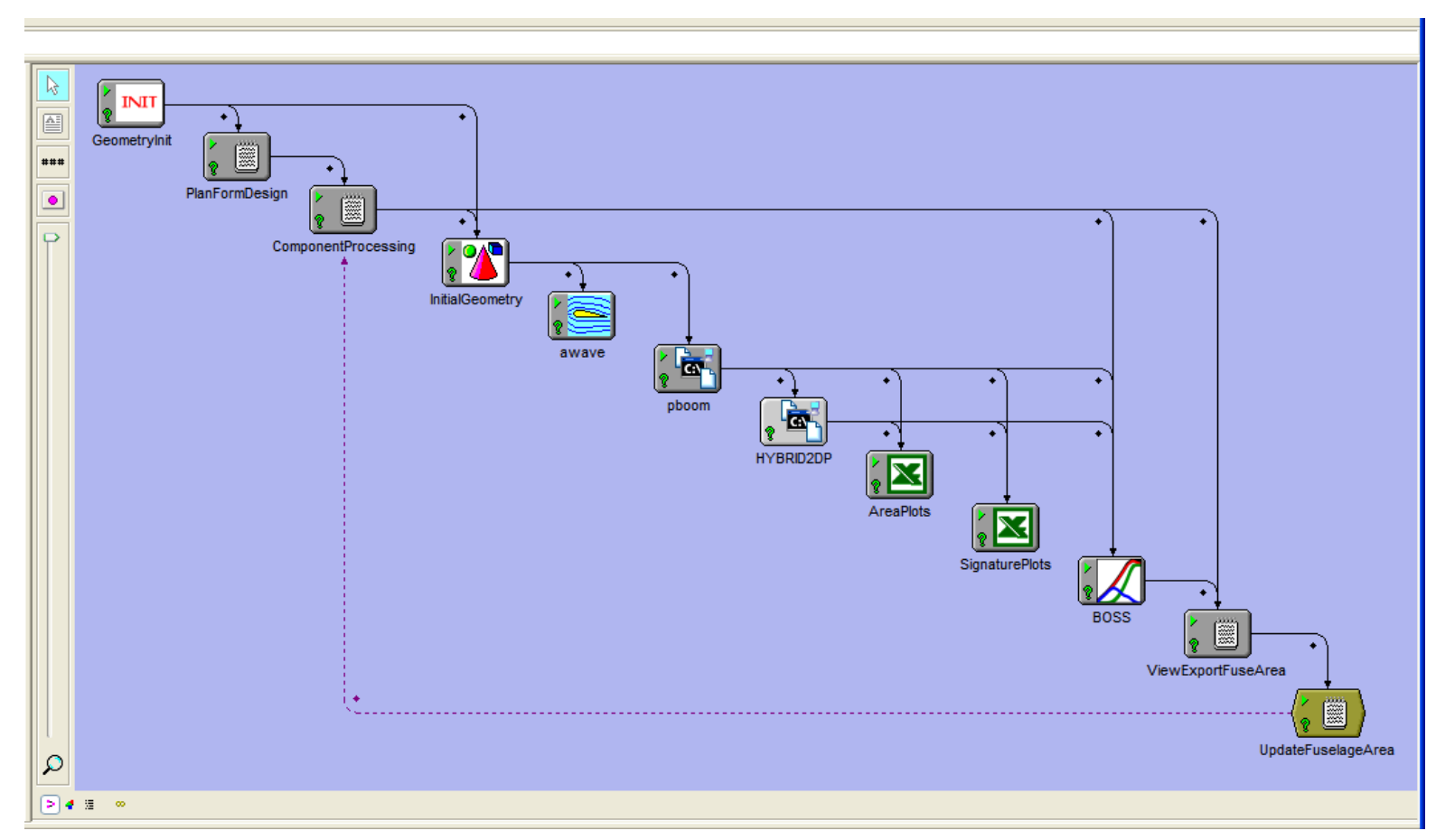

Figure 5. ModelCenter layout of the low-boom conceptual design process.

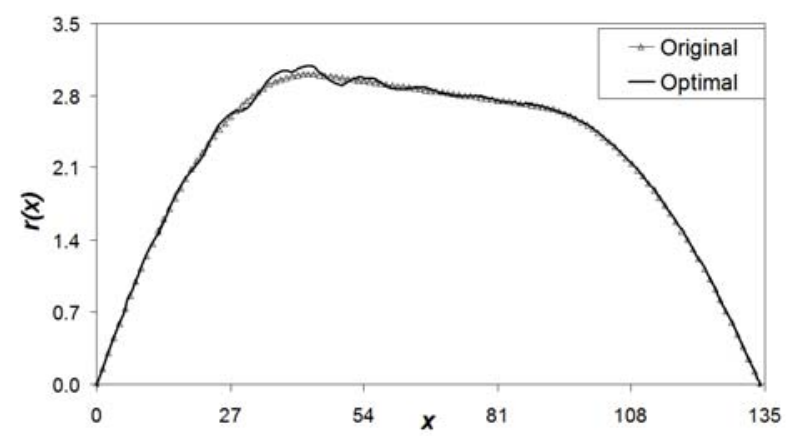

(a) Comparison of Fuselage Radii

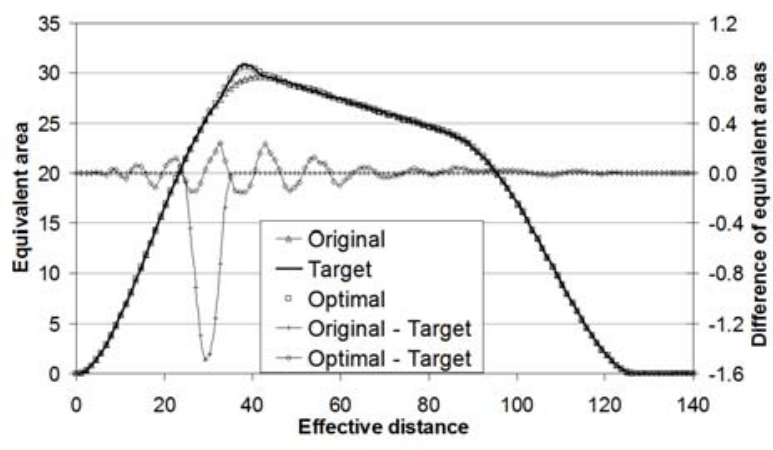

(b) Comparison of Equivalent Areas

Figure 6. Fuselage shapes and equivalent area distributions for the oscillatory case.

The oscillations are intrinsic in this case. To match the target equivalent area distribution, the fuselage radii must increase over the range $35<x<40$, which force the radii just before $x=35$ and after $x=40$ to decrease so that the equivalent areas for these $x$ values will not change. These changes lead to a wavelike effect on the fuselage shape. (See figure 6(a).) In other words, if the difference between the current and target equivalent area distributions is a local bump, then oscillatory shape modifications may be necessary for matching the equivalent area distributions.

In the second example, the target equivalent area distribution is defined as follows: $A_{e, \text { target }}(x)=A_{e}(x)+$ $\Delta A(x)$, where $A_{e}(x)$ is the equivalent area distribution of the original fuselage, $\Delta A(x)=0.000005(x-$ $35)^{2}(x-95)^{2}$ if $35 \leq x \leq 95$ and $\Delta A(x)=0$ otherwise. The same numerical optimization code is used for matching the equivalent area distributions with two different initial guesses. The first initial guess is a fuselage for which the radius distribution is defined as $\sqrt{A_{e, \text { target }}(x) / \pi}$, and the second initial guess is the original fuselage. The two initial guesses lead to two different fuselages, labeled "Solution I" and "Solution II" in figure $7(\mathrm{a})$, for which the equivalent area distributions are in very good agreement with the target 


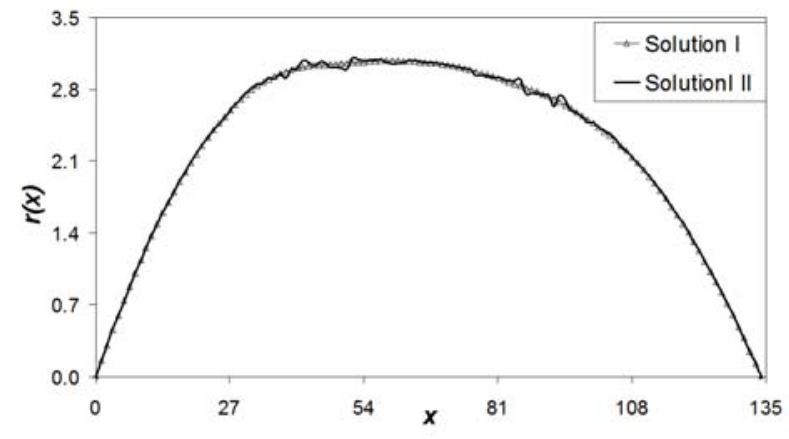

(a) Comparison of Fuselage Radii

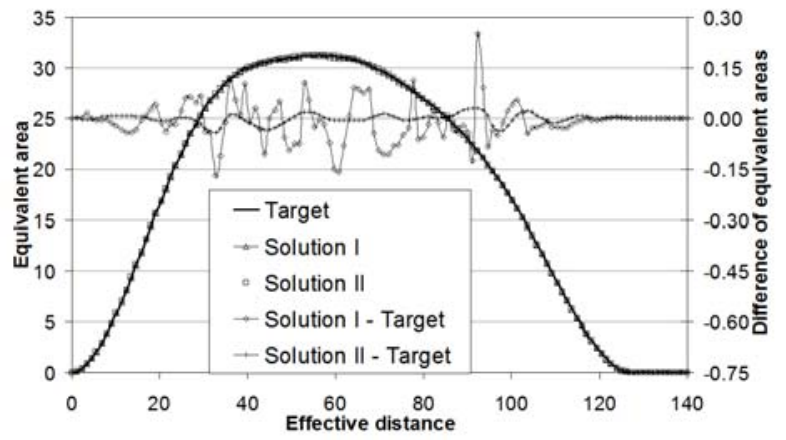

(b) Comparison of Equivalent Areas

Figure 7. Fuselage shapes and equivalent area distributions for the multiple solution case.

distribution. (See the secondary axis in figure $7(\mathrm{~b})$ for the area difference.)

The matching errors in figure 7(b) suggest that "Solution II" is not as optimal as "Solution I." However, the maximum matching error of 0.2 for "Solution II" is smaller than the 0.3 seen in the previous example. Without knowing the existence of "Solution I," one might be more than willing to accept such a good match of the equivalent area distributions. The obvious kinks in the radius distribution for "Solution II" (see figure $7(\mathrm{a})$ ) suggest that a large number of design variables for fuselage geometry modifications might lead to unnecessary oscillations in the fuselage shape. In this case, both the radius and equivalent area distributions are evaluated at 120 equally spaced locations between 0 and 135. Barger and Adams ${ }^{23}$ had a more dramatic example of one smooth and one oscillatory fuselage with the same equivalent area distribution evaluated at 27 locations. These examples indicate that the inverse problem of matching equivalent area distributions could have multiple solutions.

\section{Smoothest Shape Modifications}

Two approaches can be used to avoid the difficulties illustrated in the previous subsection. The first one is to use a spline parametric representation of the fuselage radius distribution with a few (5 to 8$)$ control points. ${ }^{29,36-39}$ For a complex configuration, such a parametric representation of fuselage radius distribution may be inadequate for fuselage shaping to match a target equivalent area distribution. In a case where a desirable fuselage shape could not be obtained, one would be left with lingering doubts whether the design space is large enough to include the right solution. In many cases, the choice of parametric forms for the design space determines whether or not a numerically optimal solution yields a meaningful design. The second approach is to allow almost arbitrary modifications of the fuselage shape, but the optimization algorithm only seeks the smoothest modification for design improvement iteratively. A successful application of this strategy has been demonstrated for transonic airfoil shape optimization. ${ }^{44}$

Note that fuselage shape change can be facilitated by a change in $r(x)$, denoted by $\Delta r(x)$. An important issue is identifying a criterion to be used for desirable design improvements during optimization iterations. For numerical minimization problems, two basic strategies determine the desirable modifications for gradientbased optimization methods. The first is based on some numerical convergence rule, and the modifications are made to achieve fast convergence to a local minimum solution (such as Newton methods). These types of methods usually do not work well for complex engineering design optimization problems as a result of numerical errors in the sensitivity calculations of those functions for which the values are generated by computer codes. The second strategy is to find a modification that achieves the greatest reduction of the objective function value in each iteration, such as the steep descent method, and terminates if no design improvement can be made. In our limited experience, this type of method only works when an appropriate parametric design space is used. Many examples can be found in literature about unrealistic but numerically optimal solutions of shape optimization problems. In practice, no mathematical model could cover all of the physics related to aircraft. System analysts are well aware that numerical optimization methods tend to generate impractical or even absurd concepts.

The smoothest shape modification ${ }^{44}$ can also be considered as an optimization strategy, based on the 
following two principles: (i) smoother shape modifications are more desirable than oscillatory ones, and (ii) smaller shape modifications are more reliable than larger ones if they have the same predicted reduction of the objective function. While the first principle doesn't require much explanation, the second one needs some justification. In general, among the feasible modifications that achieve the same predicted reduction of the objective function, the smallest shape modification has the best chance of actually reducing the value of the objective function because the prediction error of a linear Taylor approximation increases as the magnitude of the shape modifications increases. Moreover, the second principle helps to eliminate unnecessary and random shape modifications (perhaps one major reason for unrealistic optimal shapes when the number of design variables is large) and makes the modifications more relevant to desirable performance improvement based on simulation models.

To define the smoothest shape modification scheme for fuselage shape optimization, we use a cubic Bspline interpolation $z=r(x)$ of the discrete fuselage radius distribution $\left(x_{0}, r_{0}\right),\left(x_{1}, r_{1}\right), \ldots,\left(x_{n}, r_{n}\right)$, where $0=x_{0}<x_{1}<\ldots<x_{n}=l$ (the length of the fuselage). Then, fuselage shape modifications can be represented by a cubic B-spline $\Delta r(x)$ with knots at $x_{0}, x_{1}, \ldots, x_{n}$.

The third derivative $\Delta r^{(3)}(x)$ of $\Delta r(x)$ is a piecewise constant function. That is,

$$
\Delta r^{(3)}(x)=\Delta r_{i}^{(3)} \quad \text { for } x_{i-1}<x<x_{i}, \quad 1 \leq i \leq n
$$

where $\Delta r_{i}^{(3)}$ are scalars. From equation (1), one can impose an upper bound $\delta$ on $\left|\Delta r^{(3)}(x)\right|$ for all $x$ by using the following system of linear inequalities:

$$
-\delta \leq \Delta r^{(3)}\left(\frac{x_{i-1}+x_{i}}{2}\right) \leq \delta \quad \text { for } 1 \leq i \leq n
$$

Note that the radii at the fuselage nose and tail are 0. So $r\left(x_{0}\right)=r\left(x_{n}\right)=\Delta r\left(x_{0}\right)=\Delta r\left(x_{n}\right)=0$. If $\delta=0$, then $\Delta r^{(3)}(x)=0$ or $\Delta r(x)$ is a quadratic function, which results in a very smooth but not necessarily small shape modification. In fact, if $\Delta r(x)=\left(x-x_{0}\right)\left(x-x_{n}\right)$, then equation (2) is always true for any $\delta \geq 0$. Therefore, equation (2) does not control the magnitude of the shape modification. To control the magnitude of the shape modification indirectly, we add two additional constraints on the second derivative $\Delta r^{\prime \prime}(x)$ of $\Delta r(x)$ for smoothness control:

$$
-\delta \leq \Delta r^{\prime \prime}\left(x_{0}\right) \leq \delta \quad \text { and } \quad-\delta \leq \Delta r^{\prime \prime}\left(x_{n}\right) \leq \delta
$$

Then, constraints (2) and (3) with $\delta=0$ will force $\Delta r(x)=0$ because it is the only quadratic function that satisfies the conditions $\Delta r\left(x_{0}\right)=\Delta r\left(x_{n}\right)=\Delta r^{\prime \prime}\left(x_{0}\right)=\Delta r^{\prime \prime}\left(x_{n}\right)=0$. By using calculus and assuming that $l \geq 15$, one could prove that constraints (2) and (3) imply

$$
|\Delta r(x)| \leq 0.2 l^{3} \delta \text { for } 0 \leq x \leq l
$$

That is, when $\delta$ is small, constraints (2) and (3) guarantee that $\Delta r(x)$ represents a small and smooth fuselage modification.

The smoothest shape modification strategy aims to achieve a given reduction rate of the objective function by using a shape modification $\Delta r(x)$ that minimizes the maximum value of $\left|\Delta r_{i}^{(3)}\right|(0 \leq i \leq n),\left|\Delta r^{\prime \prime}\left(x_{0}\right)\right|$, and $\left|\Delta r^{\prime \prime}\left(x_{n}\right)\right|$. This strategy forces smooth shape modifications and tries to keep modified configurations as close to the existing one as possible.

\section{Fuselage Shape Optimization for Boom Minimization}

Let $A_{e, i}$ be the total equivalent area of the configuration at $x_{e, i}$, where $0=x_{e, 0}<x_{e, 1}<\ldots<x_{e, m}=l_{e}$ are the given effective distances. The total equivalent area $A_{e, i}$ includes the equivalent area due to volume and the equivalent area due to lift. (See refs. 23 and 24 for calculation of the equivalent area due to volume, and see equation (3) in ref. 45 for calculation of the equivalent area due to lift.) Let $A_{e, i}^{f}$ be the equivalent area of the fuselage volume at $x_{e, i}$ and let $A_{e, i}^{*}$ be a target equivalent area at $x_{e, i}$ for low-boom design. The design variables are fuselage radii at a given set of $x$ locations: $0=x_{0}<x_{1}<\ldots<x_{n}=l$.

The inverse design optimization problem is to find a fuselage shape such that

$$
G\left(r_{1}, \ldots, r_{n-1}\right)=\sum_{i=0}^{m}\left(A_{e, i}-A_{e, i}^{*}\right)^{2}
$$


is minimized. Unfortunately, no fuselage shape may exist that matches the target area distribution exactly (i.e., the minimum value of $G$ is not zero) or multiple fuselage shapes may exist that make $G$ almost zero. The problem is that a smaller value of $G$ does not make the corresponding ground signature closer to the target ground signature. Therefore, in the case of no exact match, the goal is to match the equivalent area distributions as closely as possible over the longest possible range starting at $x_{e}=0$. (See, for example, figure 9 of ref. 21 and figure 21 of ref. 20.)

For convenience, we use a column vector $\mathbf{r}$ to denote the design variables $r_{1}, \ldots, r_{n-1}$ and a row vector $\frac{\partial G}{\partial \mathbf{r}}$ to denote the gradient vector of partial derivatives of $G$ with respect to $r_{1}, \ldots, r_{n-1}$. Then, for any particular design vector $\mathbf{r}_{k}$, the linear Taylor polynomial approximation $L\left(\mathbf{r}_{k}, \Delta \mathbf{r}\right)$ of $G$ at $\mathbf{r}_{k}$ can be expressed as

$$
G\left(\mathbf{r}_{k}+\Delta \mathbf{r}\right)-G\left(\mathbf{r}_{k}\right) \approx L\left(\mathbf{r}_{k}, \Delta \mathbf{r}\right)=\left\langle\frac{\partial G}{\partial \mathbf{r}}\left(\mathbf{r}_{k}\right), \Delta \mathbf{r}\right\rangle
$$

After tedious calculations, one could get algebraic formulas of $G$ and $\frac{\partial G}{\partial \mathbf{r}}$.

Now we are ready to describe a scheme for the interactive inverse design optimization of the fuselage shape for low-boom supersonic concepts. This scheme, called BOSS, uses the smoothest shape modifications of the fuselage to make the equivalent area distribution move toward the target distribution interactively via some simple control parameters.

\section{Algorithm for Boom Optimization Using Smoothest Shape Modifications (BOSS)}

1. Smoothness of shape modifications. Let $\mathbf{r}_{0}$ be the current design vector. Choose a smoothness control parameter $s$ between 0 and 10, where $s=10$ means the smoothest shape modifications are used and $s=0$ means no smoothness control is used for shape modifications. The appendix describes how the smoothness control parameter $s$ is used to define the feasible set of design variables.

2. Reduction of matching errors. Inspect the differences between the equivalent area of the configuration and the target. Choose an interval of effective distances $0 \leq x_{e \text {,start }}<x_{e \text {,end }} \leq l_{e}$ and the intended reduction rate $\rho(0<\rho \leq 1)$ for the accumulated matching error $\hat{G}$ over the range $x_{e, \text { start }} \leq x_{e} \leq x_{e, \text { end }}$.

3. Selection of active design variables. Determine the largest interval $x_{\text {start }} \leq x \leq x_{\text {end }}$ such that the accumulated matching error outside of $x_{e, \text { start }} \leq x_{e} \leq x_{e, \text { end }}$ is independent of small changes of $r_{i}$ whenever $x_{i}<x_{\text {start }}$ or $x_{i}>x_{\text {end }}$.

4. Optimization iteration. For the given smoothness control parameter $s$, use an appropriate combination of the smoothest shape modification scheme and a trust region scheme to modify the design variables $r_{i}$ that correspond to $x_{\text {start }} \leq x_{i} \leq x_{\text {end }}$ for reduction of the total matching error $G$ until no further reduction is possible or $G$ is reduced by $\rho \hat{G}$.

One key idea for optimization iteration in BOSS is to couple the smoothness constraints (2) and (3) with the following trust region constraints:

$$
-\lambda_{s} \delta \leq \Delta r\left(x_{i}\right) \leq \lambda_{s} \delta \quad \text { for } 1 \leq i \leq n-1
$$

The scaling parameter $\lambda_{s}>0$ is determined by $s$ such that: if $s=10$, the constraints (6) are automatically satisfied whenever equations (2) and (3) hold; if $s=0$, the constraints (2) and (3) are automatically satisfied whenever equation (6) holds; in general, the value of $\lambda_{s}$ aims to maintain the ratio of the number of active constraints in equations (2) and (3) versus the total number of active constraints as close as possible to $s / 10$. It is a heuristic rule to transition from the smoothest shape modification scheme to a standard trust region scheme. The exact calculation of $\lambda_{s}$ is given in the appendix.

The selection of active design variables means no change of inactive design variables:

$$
\Delta r\left(x_{i}\right)=0 \text { for } x_{i}<x_{\text {start }} \text { or } x_{i}>x_{\text {end }}
$$

The optimization iteration process is to solve a sequence of subproblems that minimize the linear approximation $L\left(\mathbf{r}_{k}, \Delta \mathbf{r}\right)$ under constraints in equations (2), (3), (6), and (7). However, instead of using numerical convergence rules, we use an objective function reduction rule to control the iterations.

The waiting time for a BOSS solution is indirectly controlled by users via two easy-to-understand control parameters: $\rho$ and $s$. Obviously, if $\rho$ is smaller, then the goal of the reduction of the objective function by 
$\rho \hat{G}$ is easier to achieve; moreover, if less restriction is imposed on the smoothness of $\Delta r(x)$ (i.e., a smaller value of $s$ is used), then the reduction goal is also easier to achieve. A maximum waiting time of about three minutes is also implemented by setting a default value of 200 for the maximum number of iterations. As a result, BOSS terminates quickly with either a desirable solution or the best solution possible. This implementation allows a user to experiment with various choices of $x_{e, \text { start }}, x_{e, \text { end }}, \rho$, and $s$ to close the gap interactively between the configuration's area distribution and the target. If an optimization iteration fails to find a desirable solution, then either no further improvement could be made in the specified range or any further reduction of $G$ may require the use of less smooth fuselage shapes. The main usage of $\rho$ is to reduce potential derivative bumps near the end points $x_{\text {start }}$ and $x_{\text {end }}$ of the active design variables. The smoothness constraints in equation (2) usually do not allow derivative bumps near the end points $x_{\text {start }}$ and $x_{\text {end }}$ in each iteration. However, a large value of $\rho \hat{G}$ might require many tiny derivative bumps to be accumulated during the iterations. If this happens, then one could use a reduced value of $\rho$ and run BOSS several times with different values of $x_{e, \text { start }}$ and $x_{e, \text { end }}$ sequentially to achieve the desired reduction of $G$ while avoiding any accumulation of derivative bumps. (See the appendix for the details of the optimization iteration in BOSS.)

\section{A Case Study of Low-Boom Supersonic Conceptual Design}

To demonstrate how BOSS can assist conceptual designers design low-boom supersonic concepts, in the following subsections, we document a design process for developing a low-boom supersonic concept from a low-drag supersonic concept.

\section{A. Configuration Layout Design}

A previously designed business jet configuration, shown in figure 8, was used as a starting point to develop a low-boom configuration. No consideration was given to the boom signature when this configuration was developed. It was developed to achieve the best performance, expressed as maximum range, for a given takeoff gross weight of 100,000 lb and a balanced field length of 7,000 ft. The cabin was to be equivalent to that of a Citation X and the cruise Mach number was 1.8. This configuration was designed at the conceptual level to satisfy all of the practical considerations of the various disciplines, such as aerodynamics, structures, systems, low-speed performance, stability and control, and landing gear placement.
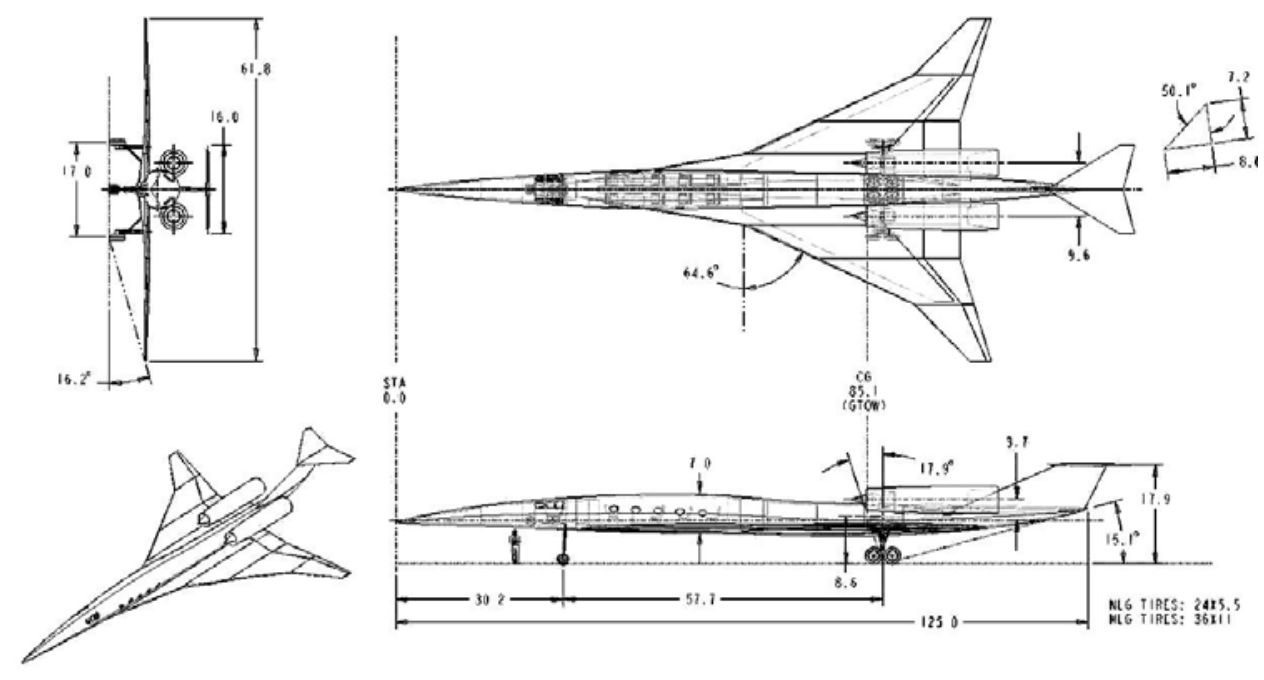

Figure 8. 3-view of a supersonic business jet concept designed for performance.

The wing planform was designed to achieve good supersonic cruise performance while maintaining adequate low-speed performance for takeoff and landing. Many studies done in conjunction with the High Speed Research Program and earlier supersonic transport studies have shown that a cranked arrow-type planform is the preferred wing planform for most supersonic cruise missions that have a significant range requirement. The high leading-edge sweep angles of the inboard portion of the wing yield a low normal leading-edge Mach 
number and an adequate lifting length for drag minimization at cruise. The size and leading-edge sweep of the outboard panels were selected to minimize induced drag and maintain adequate low-speed performance for takeoff and landing with a minimum impact on the supersonic cruise performance.

Previous studies regarding leading-edge flaps on the inboard portion of the wing have shown an insensitivity to the leading-edge camber because of the low normal Mach number on these highly swept sections. Instead, improving the low-speed performance by increasing the leading-edge radius is more effective than using leading-edge flaps, which add weight and complexity to the wing. Leading-edge flaps on the outboard portion of the wing could have some aerodynamic benefit, but the challenge of integrating them into the thin outboard panel at this point appears to override any benefit. Trailing-edge flaps are used over the complete span because they can enhance the low-speed performance of the wing significantly. The trailing-edge flaps can also be used asymmetrically for roll control.

Aggressive use of fuselage area ruling was used to minimize wave drag. This was possible because the cabin area terminates just past the beginning of the main wing area. Fuel is carried in both the fuselage and wing. A significant portion of the fuel is carried in the fuselage aft of the cabin area. A T-tail arrangement is used for the vertical and horizontal tails mainly to minimize the blocking of airflow over the tails by the wing at takeoff and landing angles of attack. The all-moving horizontal tail was sized for pitch control during takeoff and landing, and the vertical tail was sized using statistical data for engine-out conditions. The nacelles are located on the aft upper portion of the fuselage. This location is advantageous because it provides some noise shielding and alleviates the risk of foreign-object ingestion during takeoff and landing.

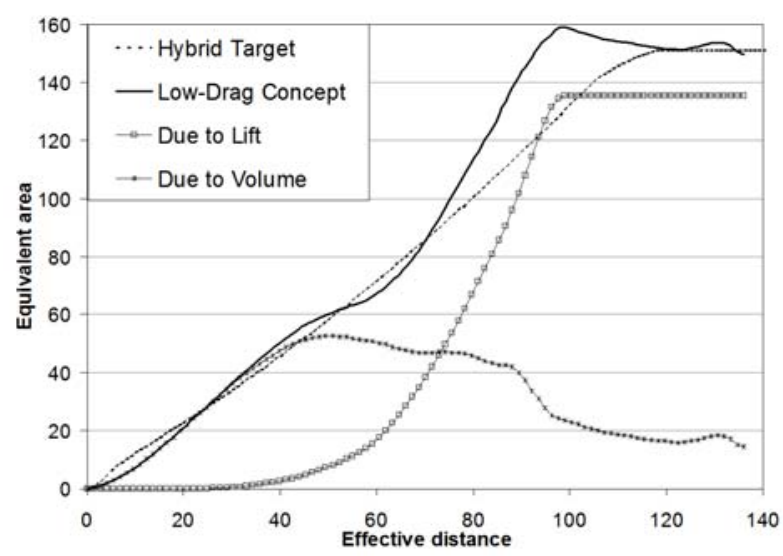

(a) Comparison of Equivalent Areas

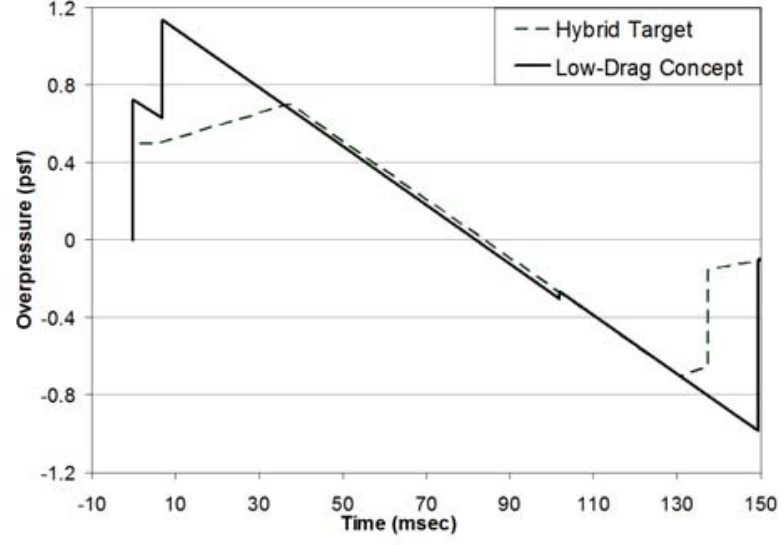

(b) Comparison of Ground Signatures

Figure 9. Equivalent area distribution and ground signature of a low-drag supersonic concept.

\section{B. Low-Boom Design by Fuselage Shaping}

The boom signature and the total equivalent area distribution of the performance configuration were analyzed using the ModelCenter model and compared with a hybrid low-boom ground signature (with an initial overpressure of $0.5 \mathrm{psf}$ ) and the corresponding total equivalent area distribution. Because no actual guidelines exist for defining an acceptable low-boom signature at this time, the hybrid signature is chosen as an acceptable target because it appears to allow for a more viable configuration than a flattop signature. The difficulty in using a flattop design is discussed later. The total equivalent area distribution that corresponds to this ground signature is calculated using the HYBRID code, which is also included in the ModelCenter model. Figure 9(a) illustrates the significant differences between the total equivalent areas for the performance configuration and the target. As stated in much of the literature that relates to low-boom design, the nose shape is critical to a shaped signature; therefore, the configuration area must match the target equivalent area closely near the nose. The other significant differences over the rest of the configuration can also have profound impact on the signature. The resulting boom signature is shown in figure $9(\mathrm{~b})$. Although not exactly an $\mathrm{N}$-wave, the peak of approximately $1.1 \mathrm{psf}$ is only a few milliseconds after the initial overpressure, and the smallest differences in flight conditions will result in the typical $\mathrm{N}$-wave that is seen in virtually all supersonic configurations not designed specifically for a low-boom requirement. 


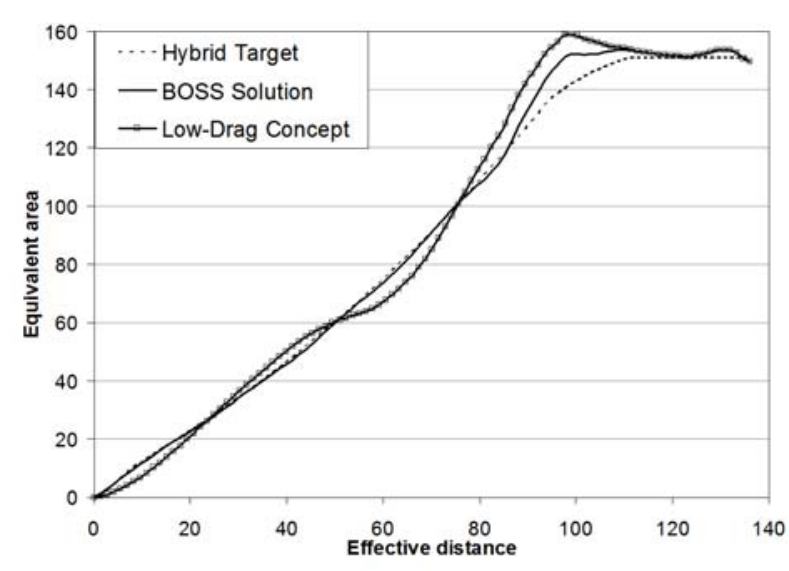

(a) Comparison of Equivalent Areas

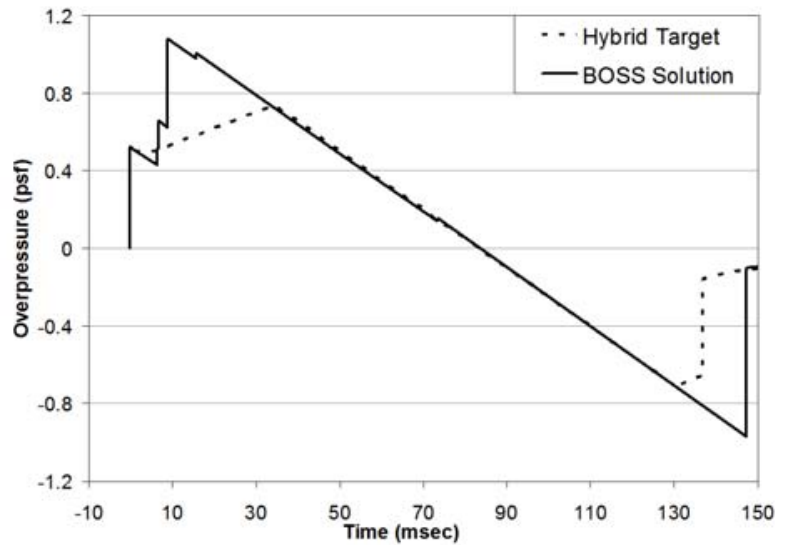

(b) Comparison of Ground Signatures

Figure 10. Use of BOSS to obtain a low-boom concept by modifying the fuselage radii of the low-drag concept.

The BOSS tool was used next to modify the fuselage areas to reduce the differences between the target and design equivalent areas. The BOSS tool allows significant flexibility in the way it can be used. In this case, the assumption was made that significant changes in the fuselage areas could be tolerated. The range over which BOSS was used was from 0 to $120 \mathrm{ft}$, in an effort to match the target equivalent area curve using almost the complete fuselage length. The smoothness control was set at fairly smooth $(s=7)$ and the reduction rate $\rho$ was set at 55 percent. Figure 10(a) shows that for approximately 60 percent of the effective distance range, a reasonable fit to the target can be accomplished. For the remaining portion of the effective distance range, the fuselage cross-section areas have decreased to near zero (see figure 11), but the corresponding equivalent areas do not match the target curve (see figure 10(a)). BOSS, therefore, quickly reveals that developing a feasible low-boom configuration by fuselage shaping alone would be extremely difficult. Furthermore, figure 10(b) shows that the signature for such a configuration would not show any signature shaping benefit. This exercise also points out that a well-designed performance-based wing planform is probably not feasible for use on a low-boom configuration. Modifications to the planform are necessary to increase the lifting length of the configuration and distribute the lift of the configuration in a manner that allows for sufficient volume for the fuselage. Changes to the wing planform primarily include higher leading-edge sweep angles for all segments of the wing. These changes could actually enhance the cruise performance of the configuration if the optimum camber shape could be used, but this enhancement comes at the expense of low-speed performance, which is critical to takeoff and landing, and may result in a range penalty for the overall configuration.

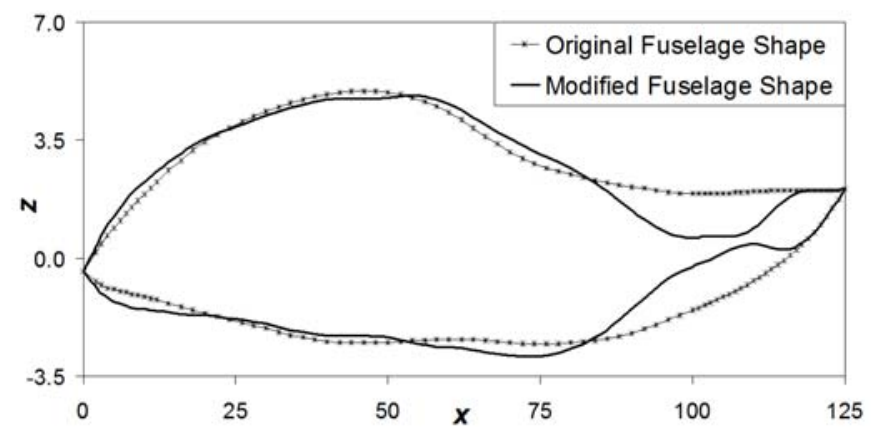

Figure 11. Side view of the low-drag fuselage shape and the modified fuselage shape generated by BOSS.

The planform modifications are outside the scope of this paper but are nonetheless necessary to obtain a viable low-boom configuration. Some experience in low-boom supersonic configuration design is desirable to make these changes. The ModelCenter model includes a component called PlanformDesign, which allows 
some changes to be made easily to the original wing planform. This component was used in an iterative manner with both PBOOM and the signature calculation components to change the wing planform until enough volume could be included in the fuselage to develop a viable configuration. The fuselage was also lengthened by $5 \mathrm{ft}$ so that more fuselage area was available to meet the target equivalent area curve and to make trimming and rotation easier. The ModelCenter model also includes a component, called ComponentProcessing, for smoothing and changing fuselage shapes. ComponentProcessing was used to modify the fuselage camber to more closely follow the camber shape of the inboard portion of the low-boom wing.

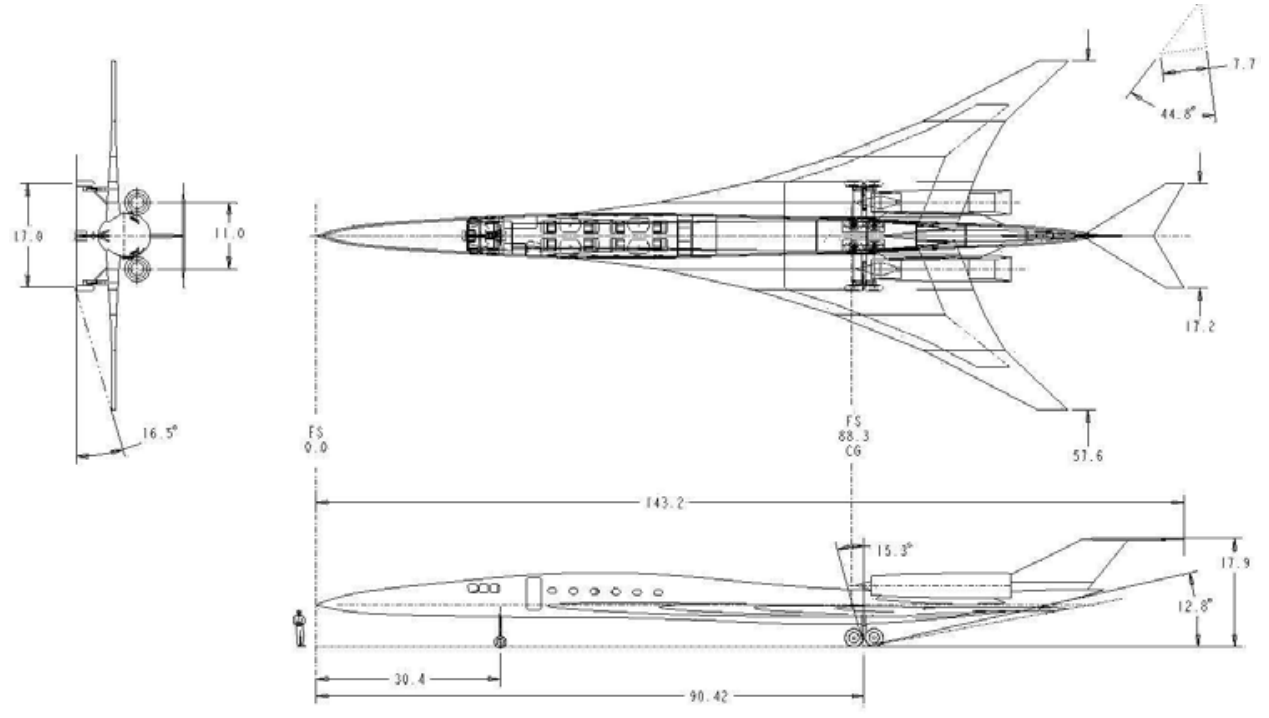

Figure 12. 3-view of a low-boom supersonic business jet concept.

After these modifications are completed, the BOSS component is used to modify the fuselage radii for quick closure to a configuration that has a smooth fuselage shape with an acceptable low-boom ground signature. Because significant differences exist between the current equivalent area distribution and the target, in the first pass through BOSS, the range over which modifications could be made is set from $x_{e, \text { start }}=0$ to $x_{e, \text { end }}=120 \mathrm{ft}$, the reduction rate $\rho$ is set high at 80 percent, and the criteria for smoothness was set at fairly smooth shape $(s=7)$. These settings allow the fuselage area distribution to rapidly approach the target over a significant portion of the effective distance range. The results are fed back into the ComponentProcessing program, where smoothing and changes to the fuselage camber line or area distribution can be made. In the second pass through BOSS, fitting is done on only the first $20 \mathrm{ft}$ of the configuration because the nose shape is critical to shaping the signature. This process of fitting the area distribution to the target using BOSS and then changing and smoothing the fuselage shape with ComponentProcessing is usually done several times until the fuselage area distribution and resulting boom signature are acceptable. Modifying the fuselage radii to match a target equivalent area distribution has in the past been one of the more time-consuming tasks in the low-boom supersonic conceptual design process. BOSS not only accomplishes this task much faster but also, when used in conjunction with the smoothing capability in the ComponentProcessing program, allows for a fuselage area distribution that can be smooth in the second derivative. This is a feature that is highly desirable when attempting to achieve an acceptable low-boom signature. Figure 12 shows the final low-boom configuration. While the resulting ground signature resembles the target signature pattern (see figure 13(b)), the differences between the equivalent area distributions are quite visible after $x_{e}=50 \mathrm{ft}$. (See figure 13(a).)

The final equivalent area distribution in figure 13(a) is a low-boom area distribution derived by a designer, via a trial-and-error approach using planform modifications and fuselage shaping in succession, to best match the "unmatchable" target area distribution. This process usually takes several days to complete. A more desirable but unavailable method is to generate a target area distribution for a low-boom ground signature that is known a priori to be realizable by a credible supersonic concept with nacelles and tails.

Note that BOSS can be used to shape the fuselage for an almost perfect match of the equivalent areas. As an illustration, we run BOSS seven times with $x_{e, \text { start }}=0, x_{e, \text { end }}=130 \mathrm{ft}$, and the reduction rate $\rho=30$ 


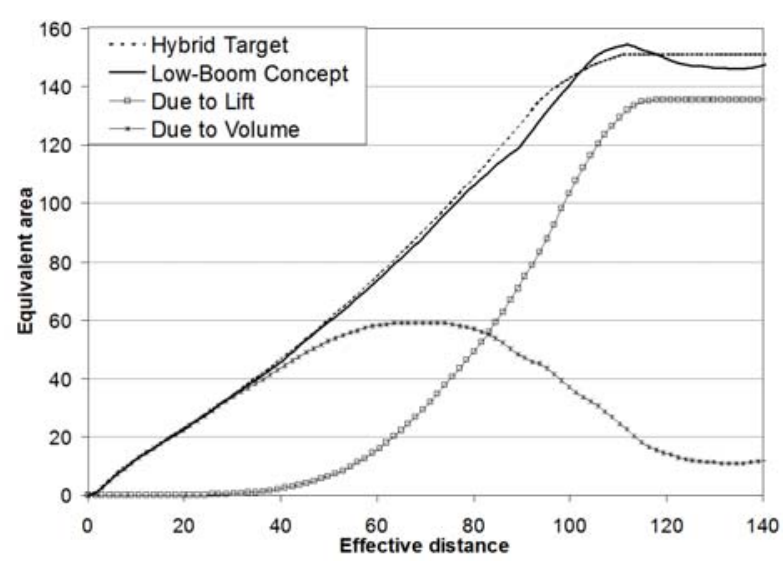

(a) Comparison of Equivalent Areas

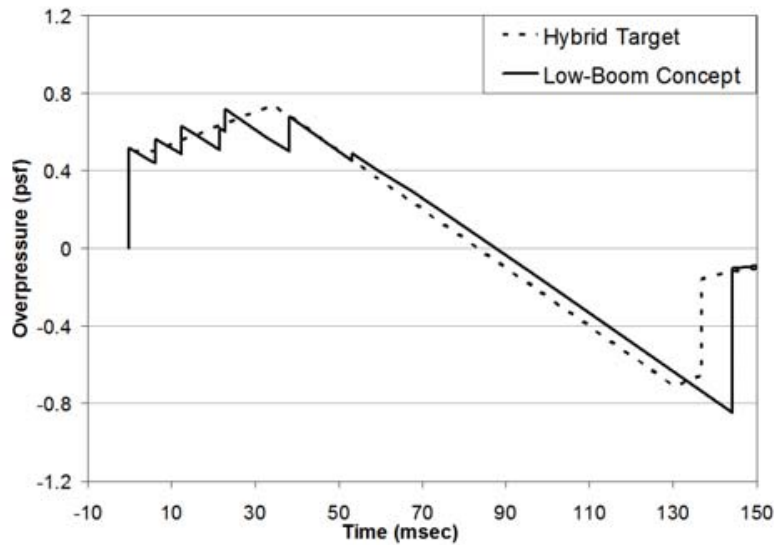

(b) Comparison of Ground Signatures

Figure 13. Equivalent area distribution and ground signature of a low-boom concept.

percent after obtaining the low-boom fuselage. The resulting numerically optimum fuselage is shown in figure 14, with the corresponding area distribution and signature plotted in figures 15(a) and 15(b), respectively. The smoothness control parameter $(s)$ for each BOSS run is set to the highest value, from among $2,3,4$, and 5, which allows a successful termination of BOSS. From the secondary axis in figure 15(a), one can see that the absolute difference between the equivalent area of the numerically optimum concept and the target is less than 0.1 up to $x_{e}=100 \mathrm{ft}$. However, figure 14 shows that the numerically optimum fuselage shape is oscillatory and cannot be considered as a credible design. Thus, BOSS is capable of providing a numerically optimum solution to the inverse design problem in most cases, but the difficult part is finding a nearly optimal solution that can be considered as a credible conceptual design.

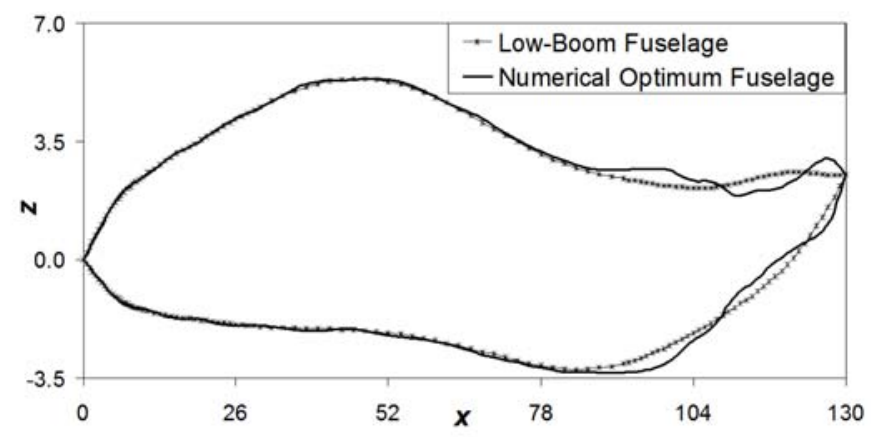

Figure 14. Side view of low-boom fuselage and numerically optimum fuselage.

\section{Trades Between Low Boom and Aerodynamic Efficiency}

The decision to not further reduce the differences between the two equivalent area distributions shown in figure 13(a) was also influenced by aerodynamic performance considerations. Adding additional area to the aft body of the fuselage was beginning to produce significant increases in the wave drag. A closer match in this area was not necessary to attain the initial overpressure desired, and any further wave drag increase would have an appreciable adverse effect on the performance of the configuration. It was decided that the configuration without the additional area was the best compromise between an acceptable low-boom signature and the performance of the configuration while still satisfying the other practical constraints that are deemed necessary. This type of trade-off is difficult to balance in a rigorous manner when designing a low-boom supersonic configuration at the conceptual level. Efforts to use purely numerical optimization 


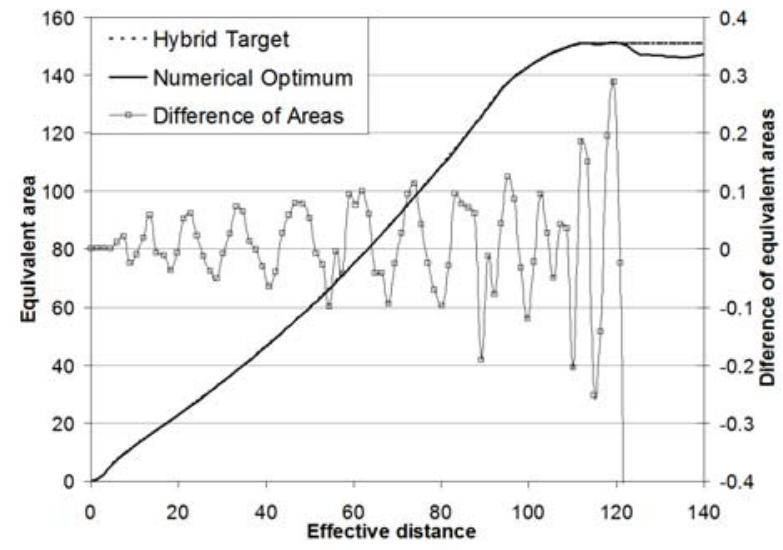

(a) Comparison of Equivalent Areas

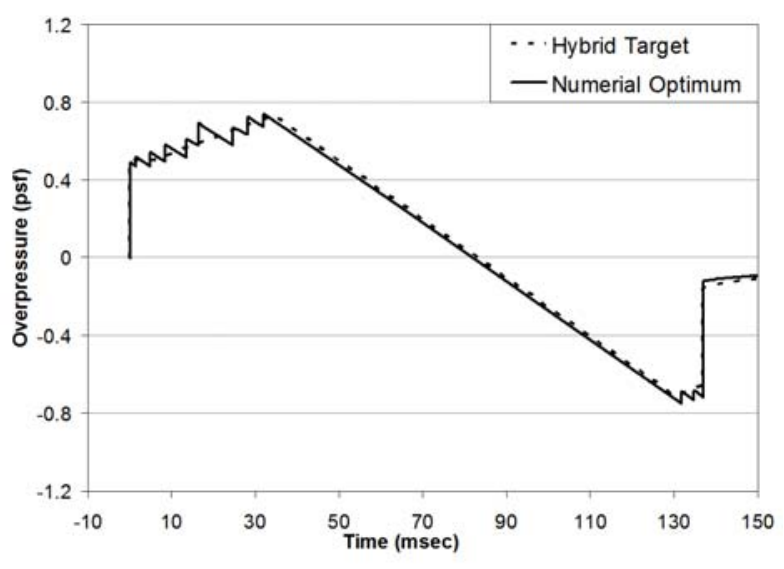

(b) Comparison of Ground Signatures

Figure 15. Equivalent area distribution and ground signature of a numerically optimum concept.

methods usually lead to dubious configurations as a result of the lack of meaningful mathematical definitions for some of the practical constraints used by designers.

Performance of the low-boom configuration was calculated based on an initial gross takeoff weight of 100,000 lb. Low-speed and high-speed polars were calculated using the modified linear methods WINGDES and AERO2S, ${ }^{46}$ and wave drag was calculated using AWAVE. ${ }^{40}$ These data, along with the geometric properties of the configuration, were entered in the sizing and performance code FLOPS. ${ }^{47}$ Sizing was handled by holding the gross takeoff weight at 100,000 lb, while thrust and wing area were the design variables. The primary constraints were takeoff and landing field length, with takeoff field length being the most critical. The modifications of the fuselage shape and wing planform to achieve a viable low-boom configuration decreased the low-speed aerodynamic performance; thus, the engine size had to increase to meet the takeoff constraint. To a lesser extent, the fuselage shaping for low boom also increased the wave drag, which had an adverse impact on the cruise portion of the mission. The overall impact of the changes that were made to create a viable low-boom configuration resulted in a decrease in range from 3,500 n.m. to 3,000 n.m., or about a 15 percent decrease in performance when measured in this manner. A fruitful area for further design refinement is how to modify the configuration to minimize this penalty and maintain an acceptable low-boom signature.

\section{Effects of Target Ground Signatures}

After a viable low-boom configuration is obtained, studies can be completed quickly to determine how variations in the target signature would impact the configuration. One such variation would be to change our target signature from a hybrid to a flattop signature. Flattop signatures are less likely than the hybrid signatures to be degraded by changes in the atmosphere. The flattop target signature is generated by changing the slope parameter in the HYBRID code, which also calculates the corresponding total target equivalent area distribution. The fuselage of the configuration is then modified using BOSS to match the target equivalent area distribution, and the results are shown in figures 16(a) and 16(b). Figure 16(a) shows that BOSS matched the target equivalent area distribution quite well, and figure 16(b) shows that the signature is indeed approaching a flattop shape with only a few BOSS runs. However, figure 17 shows that the fuselage cross-section areas in the aft portion of the fuselage decrease to the extent that obtaining a viable low-boom configuration by fuselage shaping alone would be difficult. Therefore, designing a supersonic concept with a flattop signature requires either further wing planform changes or lengthening of the fuselage or both. Because these types of studies can be done quickly, many such questions about how fundamental changes to the configuration affect the viability of the configuration and the impact they have on the signature can be addressed at the conceptual design level with little effort. It is important to answer as many of these questions as possible early in the design process before more time-consuming and expensive analyses and tests are performed. 


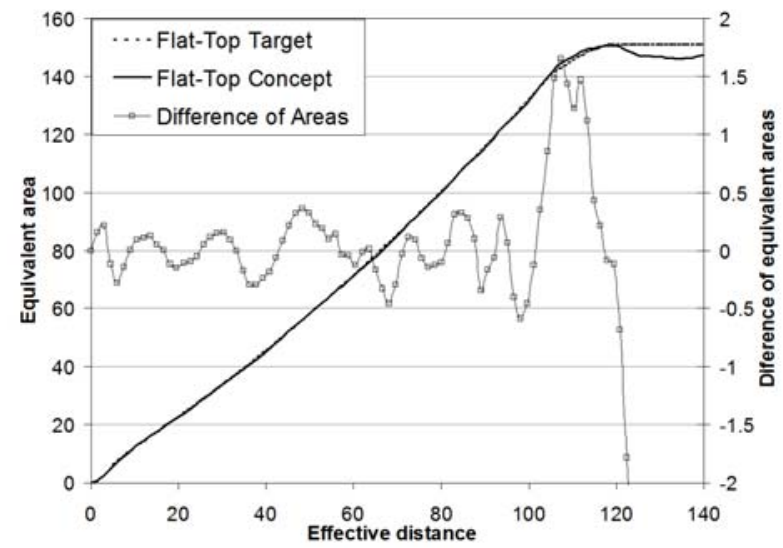

(a) Comparison of Equivalent Areas

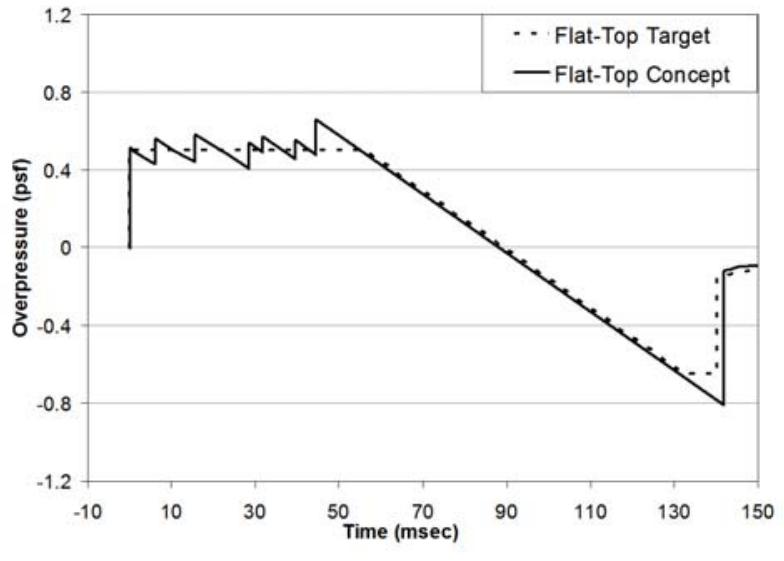

(b) Comparison of Signatures

Figure 16. Ground signature and area distribution of a flattop supersonic concept.

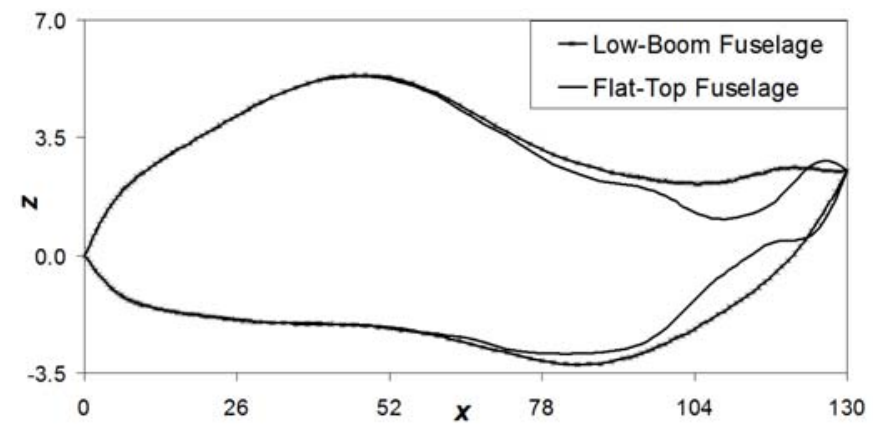

Figure 17. Side view of the fuselage for the flattop concept.

\section{Relationship Between Area Distribution and Ground Signature}

From the previous case study of low-boom conceptual design, it is clear that a target area distribution derived from the SGD theory may not be realized by a practical supersonic concept with nacelles and tails. The designer must find a configuration for which the equivalent area distribution closely matches the target in such a way that the resulting ground signature is similar to the target low-boom signature. The ground signature of the final low-boom concept may be similar to the target signature (see figure 13(b)), but the two equivalent area distributions are visibly different. (See figure 13(a).) Even with almost perfect matching of equivalent area distributions, "saw-tooth" oscillations still exist in the ground signature of the configuration. (See figures 15(a) and 15(b).) These results show two characteristics of the relationship between the equivalent area distribution and the ground signature: (i) similarly shaped ground signatures can have significantly different equivalent area distributions, and (ii) "saw-tooth" oscillations in ground signatures are unavoidable for supersonic concepts with nacelles and tails. In this section, we attempt to provide some insight into these two characteristics of the relationship between the equivalent area distribution and the boom signature.

Let $\mathcal{E}$ be the region bounded above by the maximum value and below by the minimum value of the $A_{e}$ distributions for ramp, hybrid $1, \ldots$, hybrid 4 , and flattop signatures plotted in figure 1(a). Then, the $A_{e}$ distribution for the low-boom design lies in $\mathcal{E}$ for $x_{e}<80 \mathrm{ft}$. (See figure 18.) We can conjecture that other $A_{e}$ distributions can be found in the envelope region $\mathcal{E}$ with low-boom ground signature. No general method exists to define a target $A_{e}$ distribution in $\mathcal{E}$ with a low-boom ground signature. However, one can easily construct low-boom $A_{e}$ distributions that are different from those derived by the SGD theory. 


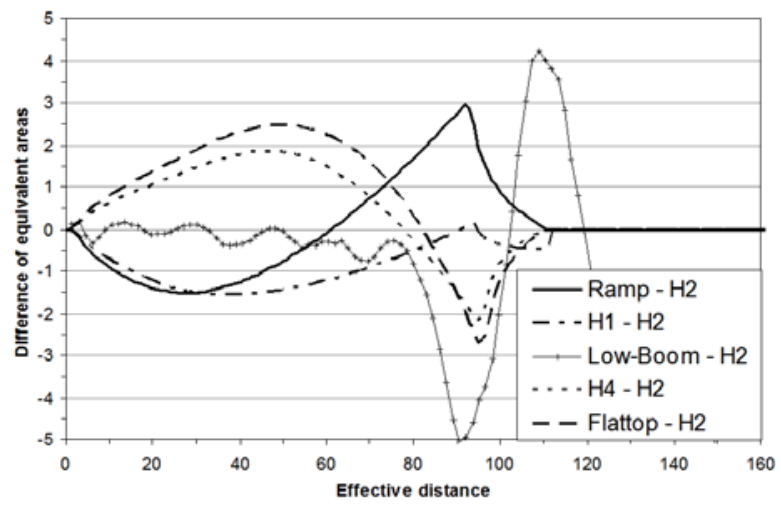

Figure 18. Deviations of other equivalent area distributions from that for Hybrid 2.

Let $A_{e, 1}\left(x_{e}\right)$ and $A_{e, 2}\left(x_{e}\right)$ be two $A_{e}$ distributions that correspond to the low-boom signatures plotted in figure $1(\mathrm{a})$. Then, the following convex combination $A_{e}^{*}\left(x_{e}\right)$ of these two $A_{e}$ distributions also has a low-boom ground signature

$$
A_{e}^{*}\left(x_{e}\right)=\tau\left(x_{e}\right) A_{e, 1}\left(x_{e}\right)+\left[1-\tau\left(x_{e}\right)\right] A_{e, 2}\left(x_{e}\right)
$$

where $\tau\left(x_{e}\right)$ is a linear polynomial with its values between 0 and 1 for $0 \leq x_{e} \leq l_{e}$. Figure 19(a) shows how the three constructed $A_{e}$ distributions differ from the $A_{e}$ distribution for the hybrid 2 (H2) signature, where $\mathrm{HiHj}$ denotes a convex combination of hybrid $\mathrm{i}$ and hybrid $\mathrm{j}$. These constructed $A_{e}$ distributions have the same characteristics as the original hybrid signatures. See figure 19(b).

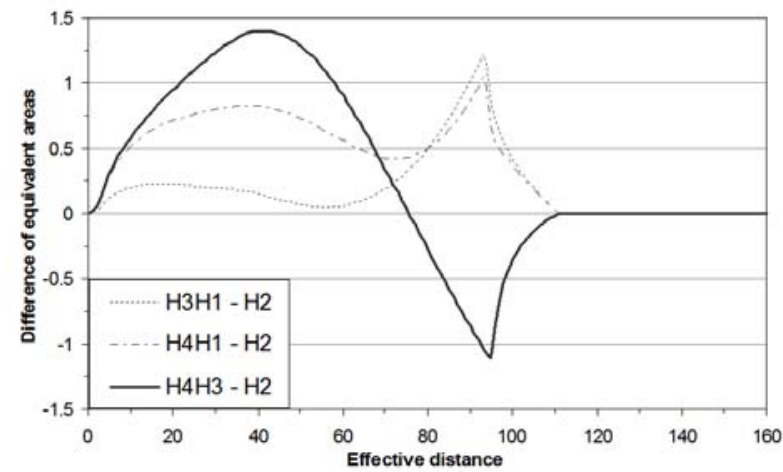

(a) Comparison of Equivalent Areas

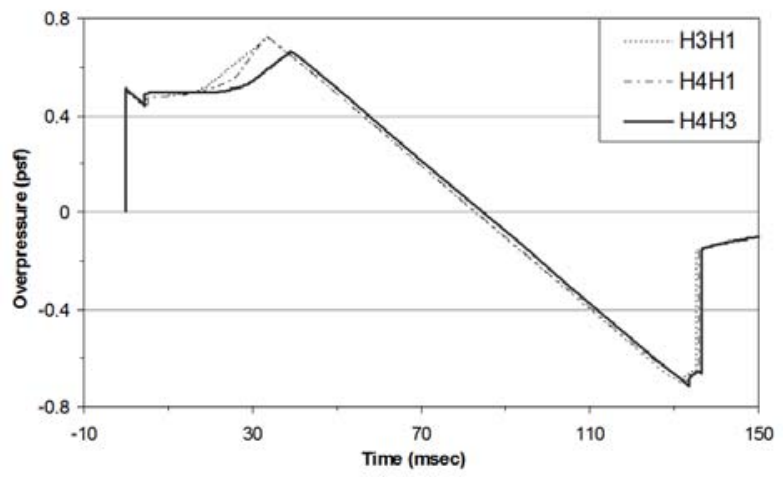

(b) Comparison of Signatures

Figure 19. Ground signature and area distribution of a flattop supersonic concept.

The need for a new target $A_{e}$ distribution usually arises when the given target becomes incompatible with the volume requirement for a practical configuration. For low-boom supersonic concept design using Whitham's boom prediction method, it is important to have a capability to increase or decrease the value of a given $A_{e}$ target over a specific $x_{e}$ range without adverse effect on the predicted ground signature. Methods that are more flexible than using convex combinations of $A_{e}$ distributions derived by the SGD theory need to be developed.

In Whitham's boom prediction theory, each vertical line segment in the boom signature is caused by shock waves. However, we do not yet understand how numerical errors in the calculation of the $A_{e}$ distribution affect the oscillations in the predicted ground signature. Note that the differences between the $A_{e}$ distribution for the low-boom design and the target are caused by both the numerical errors in the $A_{e}$ calculation and the actual mismatch of the $A_{e}$ distributions. Numerical errors in the $A_{e}$ calculation include numerical errors in the lift distribution and discretization errors in the design's volume calculation. These two sources of errors 
can not be separated. Therefore, we can only assess their cumulative effect on the ground signature. The basic method is to eliminate the matching errors over various $x_{e}$ ranges to see how the predicted ground signature changes. The process is to use a convex combination $A^{*}\left(x_{e}\right)$ of $A_{e}\left(x_{e}\right)$ of the low-boom design and the target $A_{e, \text { target }}$ defined by the following formula:

$$
A_{e}^{*}\left(x_{e}\right)=\tau\left(x_{e}\right) A_{e}\left(x_{e}\right)+\left[1-\tau\left(x_{e}\right)\right] A_{e, \text { target }}\left(x_{e}\right)
$$

where $\tau\left(x_{e}\right)$ is a piecewise linear polynomial defined by one of the following two formulas:

$$
\tau\left(x_{e}\right)=\left\{\begin{array}{ll}
0 & \text { if } x_{e} \leq x_{e}^{*} \\
\frac{x_{e}-x_{e}^{*}}{l_{e}-x_{e}^{*}} & \text { if } x_{e}>x_{e}^{*}
\end{array} \quad \text { or } \quad \tau\left(x_{e}\right)= \begin{cases}0 & \text { if } x_{e}>x_{e}^{*} \\
\frac{x_{e}-x_{e}^{*}}{l_{e}-x_{e}^{*}} & \text { if } x_{e} \leq x_{e}^{*}\end{cases}\right.
$$

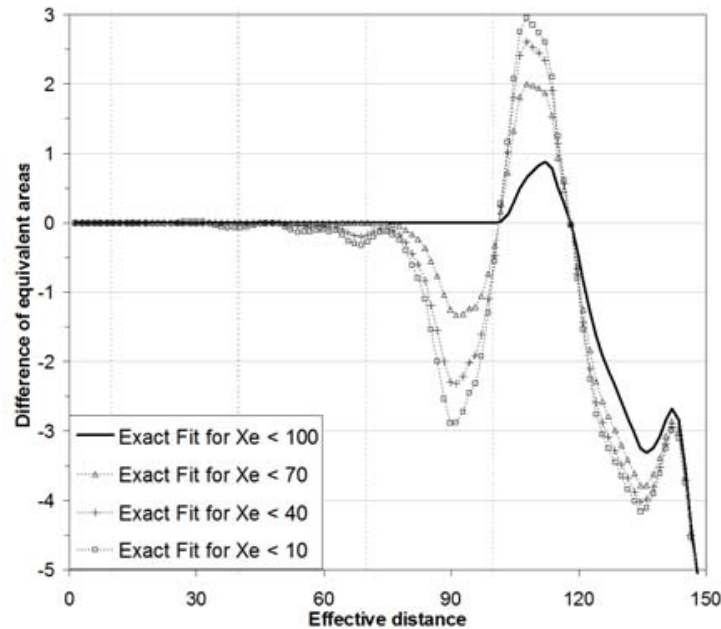

(a) Comparison of Matching Errors

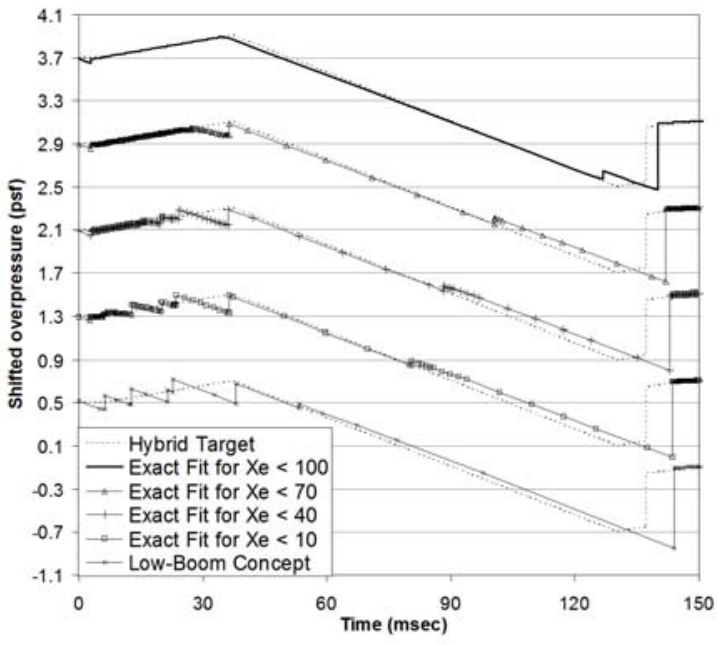

(b) Comparison of Signatures

Figure 20. Sensitivity of predicted ground signatures with respect to aft $A_{e}$ matching errors.

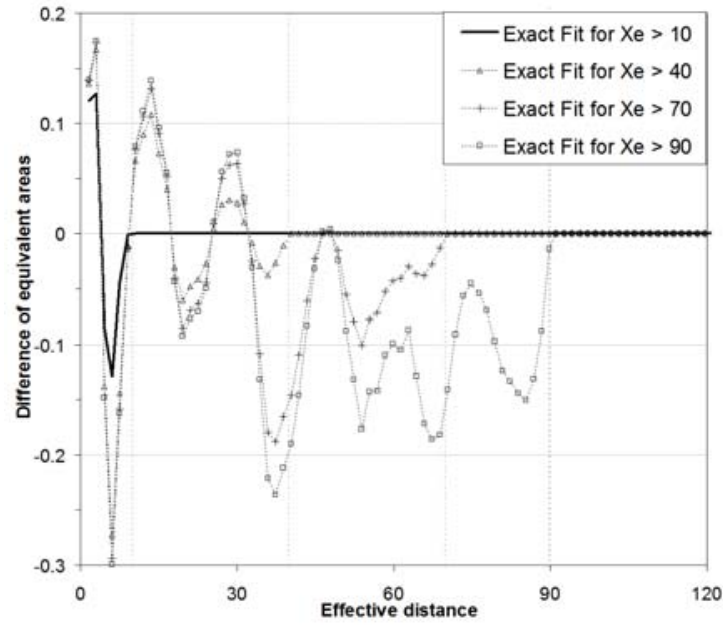

(a) Comparison of Matching Errors

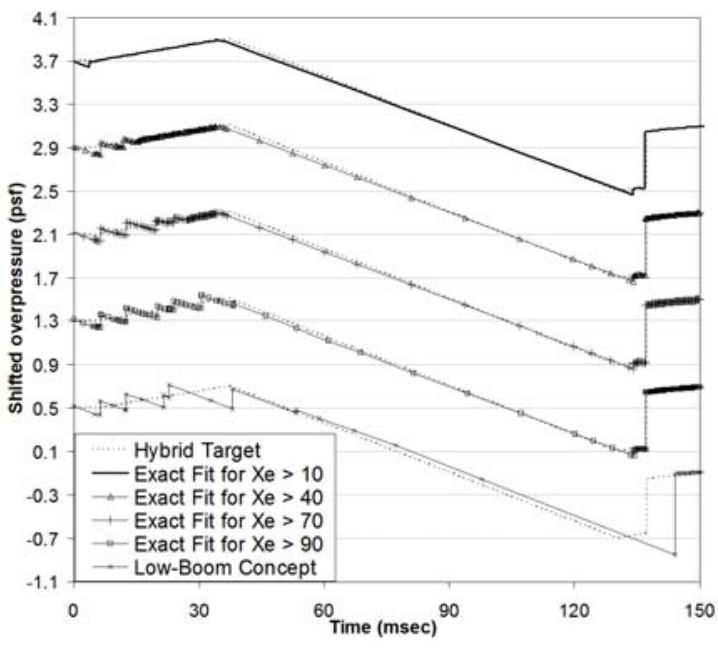

(b) Comparison of Signatures

Figure 21. Sensitivity of predicted ground signatures with respect to front $A_{e}$ matching errors.

Figure 20(a) shows the differences of $A_{e}^{*}\left(x_{e}\right)$ generated by the first formula in equation (10) and the target distribution, with $x_{e}^{*}=10,40,70$, and 100. Figure 20(b) shows how the oscillations in the front part of the 
predicted ground signature gradually disappear as the matching errors become zero for $x_{e} \leq x_{e}^{*}(=10,40,70$, and 100). Figure 21(a) shows the differences of $A_{e}^{*}\left(x_{e}\right)$ generated by the second formula in equation (10) and the target distribution, with $x_{e}^{*}=90,70,40$, and 10. Figure 21(b) shows that the aft part of the predicted ground signature matches the target signature when the matching errors become zero for $x_{e} \geq 90$, and it also shows how the oscillations in the front part of the predicted ground signature gradually disappear as the matching errors become zero for $x_{e} \geq x_{e}^{*}(=90,70,40$, and 10). In practice, achieving the perfect matching of $A_{e}$ distributions over any reasonable range of $x_{e}$ is impossible. As a result, the predicted ground signature will always contain oscillations because of its sensitivity with respect to the second derivative of the $A_{e}$ distribution.

\section{Concluding Remarks}

In this paper, we introduce an interactive design optimization tool called BOSS (Boom Optimization using Smoothest Shape modifications), for generating low-boom configurations by modifying the fuselage shape to reduce the discrepancies between the design equivalent area distribution of a supersonic concept and a target distribution derived from the boom minimization theory.

The boom prediction code based on Whitham's F-function method, PBOOM, is used for sonic-boom analysis. BOSS uses the smoothest shape modification strategy to modify the fuselage radius distribution at 100 or more longitudinal locations and to find smooth fuselage shapes that reduce the discrepancies between the design and target equivalent area distributions over any specified range of effective distance. A smoothing tool is also incorporated into the design process so that any minor oscillation in the resultant fuselage radius distribution can be easily removed with minimum change to the fuselage shape. All the tools are integrated as plug-and-play components in the ModelCenter framework.

For any given supersonic concept (with wing, fuselage, nacelles, tails, and optional canards), a designer can examine the differences between the design and target equivalent areas, decide which part of the design equivalent area curve needs to be modified, choose a desirable rate for the reduction of the discrepancies over the specified range, and select a parameter for smoothness control of fuselage shapes. Then BOSS generates a fuselage shape based on the designer's inputs in a matter of seconds. If the generated solution is not acceptable, the designer can work on a different part of the equivalent area curve, change the rate of reduction, or relax the smoothness control until a desirable solution is found. The new configuration is analyzed by PBOOM to determine whether it has an acceptable low-boom ground signature. If not, the designer can use BOSS to further reduce the differences between the design and target equivalent areas until the configuration has an acceptable low-boom ground signature. Using BOSS and PBOOM, the designer can generate a realistic, smooth fuselage shape that results in a supersonic configuration with a low-boom ground signature in a few hours. In addition, a designer can use BOSS to quickly eliminate any configuration that cannot achieve low-boom characteristics with fuselage shaping alone.

For any given wing planform and layout of aircraft components, BOSS reduces the design time of lowboom supersonic concepts from months to hours. More importantly, BOSS allows a quick closure of the fuselage shaping process because BOSS lets the designer see how much deterioration of the fuselage shape is necessary for further reduction of the discrepancies between the design and target equivalent area distributions.

A conceptual design case study demonstrates how BOSS can be used to develop a low-boom supersonic concept from a low-drag supersonic concept.

The perturbation study demonstrates that oscillations in the sonic-boom signature may be unavoidable because exact matching of the target equivalent area distribution is unlikely. In addition, the study also shows that making the target equivalent area distributions realizable by a practical configuration, while maintaining a shaped sonic-boom signature, is desirable. One approach to generating alternate equivalent area distributions is demonstrated by using convex combinations of target equivalent area distributions from the SGD theory. However, the question of whether these new equivalent area distributions could be matched by practical configurations has not been answered.

The next step is to integrate CFD analysis into the current low-boom conceptual design process to make the generated low-boom concept more credible. Also, BOSS can be extended to make it applicable to a fuselage with noncircular cross sections. 


\section{References}

${ }^{1}$ Seebass, R., and George, A., "Sonic-Boom Minimization," Journal of the Acoustical Society of America, Vol. 51, No. 2, Pt. 3, 1972, pp. 686-694.

${ }^{2}$ Darden, C., "Minimization of Sonic Boom Parameters in Real and Isothermal Atmosphere," NASA TN-D-7842, 1975.

${ }^{3}$ Darden, C., "Sonic Boom Minimization With Nose-Bluntness Relaxation," NASA TP-1348, 1979.

${ }^{4}$ Whitham, G., "The Flow Pattern of a Supersonic Projectile," Communications on Pure and Applied Mathematics, Vol. V, No. 3, 1952, pp. 301-348.

${ }^{5}$ Walkden, F., "The Shock Pattern of a Wing-Body Combination, Far From the Flight Path," Aeronautical Quarterly, Vol. IX, Pt. 2, May 1958, pp. 164-194.

${ }^{6}$ McMasters, J., and Cummings, R., "Airplane Design - Past, Present, and Future," Journal of Aircraft, Vol. 39, No. 1, 2002, pp. 10-17.

${ }^{7}$ Seebass, R., and Argow, B., "Sonic Boom Minimization Revisited," AIAA 1998-2956, The 2nd Theoretical Fluid Mechanics Meeting, June 1998.

${ }^{8}$ Morgenstern, J., "Low Sonic Boom Design and Performance of a Mach 2.4/1.8 Overland High Speed Civil Transport," in High-Speed Research: Sonic Boom, Volume II, edited by C. Darden, NASA-CP-3173, Proceedings of a conference held at Langley Research Center, Hampton, Virginia, Feb. 1992, pp. 55-63.

${ }^{9}$ Haglund, G., and Ogg, S., "Two HSCT Mach 1.7 Low Boom Designs," in High-Speed Research: Sonic Boom, Volume II, edited by C. Darden, NASA-CP-3173, Proceedings of a conference held at Langley Research Center, Hampton, Virginia, Feb. 1992, pp. 65-87.

${ }^{10}$ Makino, Y., Sugiura, T., Watanuki, T., Kubota, H., Aoyama, T., and Iwamiya, T., "The Effect of Nose Bluntness of a Low-Boom Configuration on Sonic-Boom," AIAA-1997-2213, The 15th AIAA Applied Aerodynamics Conference, Atlanta, GA, June 1997.

${ }^{11}$ Makino, Y., Suzuki, K., Noguchi, M., and Yoshida, K., "Non-Axisymmetrical Fuselage Shape Modification for Drag Reduction of a Low Sonic-Boom Airplane," AIAA-2003-557, 41st Aerospace Sciences Meeting and Exhibit, Reno, Nevada, Jan. 2003.

${ }^{12}$ Makino, Y., Suzuki, K., Noguchi, M., and Yoshida, K., "Nonaxisymmetrical Fuselage Shape Modification for Drag Reduction of Low-Sonic-Boom Airplane," AIAA Journal, 2003, Vol. 41, No. 8, pp. 1413-1420.

${ }^{13}$ Sasaki, D., and Obayashi, S., "Low-Boom Design Optimization for SST Canard-Wing-Fuselage Configuration," AIAA2003-3432, The 16th AIAA Computational Fluid Dynamics Conference, Orlando, Florida, June 2003.

${ }^{14}$ Graham, D., Dahlin, J., Meredith, K., and Vadnais, J., "Aerodynamic Design of Shaped Sonic Boom Demonstration Aircraft," AIAA-2005-8, The 43rd AIAA Aerospace Sciences Meeting and Exhibit, Reno, Nevada, Jan. 2005.

${ }^{15}$ Haglund, G., "High Speed Civil Transport Design for Reduced Boom," Boeing Document No. D6-55430, NASA Contract No. NAS1-18377, 1991.

${ }^{16}$ Mack, R., and Haglund, G., "A Practical Low-Boom Overpressure Signature Based on Minimum Sonic Boom Theory," in High-Speed Research: Sonic Boom, Volume II, edited by C. Darden, NASA-CP-3173, Proceedings of a conference held at Langley Research Center, Hampton, Virginia, Feb. 1992, pp. 15-30.

${ }^{17}$ Mack, R., and Needleman, K., "A Methodology for Designing Aircraft to Low Sonic Boom Constraints," NASA TM-4246, 1991.

${ }^{18}$ Mack, R., "Additional F-Functions Useful for Preliminary Design of Shaped-Signature, Low-Boom, Supersonic-Cruise Aircraft," NASA CP-209699, Dec. 1999. 1991.

${ }^{19}$ Shepard, K., and Sullivan, B., "A Loudness Calculation Procedure Applied to Shaped Sonic Booms," NASA TP-3134,

${ }^{20}$ Aronstein, D., and Schueler, K., "Two Supersonic Business Aircraft Conceptual Designs With and Without Sonic Boom Constraint," Journal of Aircraft, Vol. 42, No. 3, 2005, pp. 775-786.

${ }^{21}$ Mack, R., "A Supersonic Business-Jet Concept Designed for Low Sonic Boom," NASA TM-212435, 2003.

${ }^{22}$ Choi, S., Alonso, J., and Weide, E., "Numerical and Mesh Resolution Requirements for Accurate Sonic Boom Prediction of Complete Aircraft Configurations," AIAA-2004-1060, The 42nd AIAA Aerospace Sciences Meeting and Exhibit, Reno, Nevada, Jan. 2004.

${ }^{23}$ Barger, R., and Adams, M., "Fuselage Design for a Specified Mach-Sliced Area Distribution," NASA TP-2975, 1990.

${ }^{24}$ Mack, R., and Needleman, K., "A Semiempirical Method for Obtaining Fuselage Normal Areas From Fuselage Mach Sliced Area," NASA TM-4228, 1990.

${ }^{25}$ Rallabhandi, S., and Mavris, D., "Sonic Boom Minimization Using Inverse Design and Probabilistic Acoustic Propagation," Journal of Aircraft, Vol. 43, No. 6, 2006, pp. 1815-1828.

${ }^{26}$ Darden, C., "Limitations of linear theory for sonic boom calculations," Journal of Aircraft, Vol. 30, No. 3, 1993, pp. 309-314.

${ }^{27}$ Thomas, C., "Extrapolation of Sonic Boom Pressure Signatures by the Waveform Parameter Method," NASA TN D-6832, June 1972 .

${ }^{28}$ Plotkin, K., "PCBoom3 Sonic Boom Prediction Model - Version 1.0e," Wyle Research Report WR 95-22E, Oct. 1998.

${ }^{29}$ Makino, Y., Aoyama, T., Iwamiya, T., Watanuki, T., and Kubota, H., "Numerical Optimization of Fuselage Geometry to Modify Sonic-Boom Signature," Journal of Aircraft, 1999, Vol. 36, No. 4, pp. 668-674.

${ }^{30}$ Howe, D., "Sonic Boom Reduction Through the Use of Non-Axisymmetric Configuration Shaping," AIAA-2003-929, The 41st Aerospace Sciences Meeting and Exhibit, Reno, Nevada, Jan. 2003.

${ }^{31}$ Nadarajah, S., Jameson, A., and Alonso, J., "Sonic Boom Reduction Using an Adjoint Method for Wing-Body Configurations in Supersonic Flow," AIAA-2002-5547, 9th AIAA/ISSMO Symposium on Multidisciplinary Analysis and Optimization, Atlanta, Georgia, Sep. 2002. 
${ }^{32}$ Morgenstern, J., Arslan, A., Lyman, V., and Vadyak, J., "F-5 Shaped Sonic Boom Demonstrator's Persistence of Boom Shaping Reduction through Turbulence," AIAA-2005-0012, The 43rd AIAA Aerospace Sciences Meeting and Exhibit, Reno, Nevada, Jan. 2005.

${ }^{33}$ Pawlowski, J., Graham, D., Boccadoro, C., Coen, P., and Maglieri, D., "Origins and Overview of the Shaped Sonic Boom Demonstration Program," AIAA-2005-5, The 43rd AIAA Aerospace Sciences Meeting and Exhibit, Reno, Nevada, Jan. 2005.

${ }^{34}$ Farhat, C., Maute, K., Argrow, B., and Nikbay, M., "A Shape Optimization Methodology for Reducing the Sonic Boom Initial Pressure Rise," AIAA-2002-145, The 40th AIAA Aerospace Sciences Meeting and Exhibit, Reno, NV, Jan. 2002.

35 Alonso, J., Kroo, I., and Jameson, A., "Advanced Algorithms for Design and Optimization of Quiet Supersonic Platforms," AIAA-2002-0144, The 40th AIAA Aerospace Sciences Meeting and Exhibit, Reno, Nevada, Jan. 2002.

${ }^{36}$ Choi, S., Chung, H., and Alonso, J., "Design of Low-Boom Supersonic Business Jet with Evolutionary Algorithms Using Adaptive Unstructured Mesh Method," AIAA-2004-1758, 45th AIAA/ASME/ASCE/AHS/ASC Structures, Structural Dynamics and Materials Conference, Palm Springs, California, Apr. 2004.

${ }^{37}$ Choi, S., Alonso, J., Kroo, I., and Wintzer, M., "Multi-fidelity Design Optimization of Low-Boom Supersonic Business Jets," AIAA-2004-4371, 10th AIAA/ISSMO Multidisciplinary Analysis and Optimization Conference, Albany, NY, Aug. 2004.

${ }^{38}$ Choi, S., Alonso, J., and Kroo, I., "Multi-Fidelity Design Optimization Studies for Supersonic Jets Using Surrogate Management Frame Method," AIAA-2005-5077, 23rd AIAA Applied Aerodynamics Conference, Toronto, Ontario, June 2005.

${ }^{39}$ Makino, Y., and Kroo, I., "Robust Objective Functions for Sonic-Boom Minimization," Journal of Aircraft, Vol. 43, No. 5, 2006, pp. 1301-1305.

${ }^{40}$ Harris, R., Jr., "An Analysis and Correlation of Aircraft Wave Drag," NASA TM-X-947, March 1964.

${ }^{41}$ Carlson, H., and Mack, R., "Estimation of Wing Nonlinear Aerodynamic Characteristics at Supersonic Speeds," NASA TP-1718, 1986

${ }^{42}$ Coen, P., "Development of a Computer Technique for the Prediction of Transport Aircraft Flight Profile Sonic Boom Signatures," M.S. Thesis, School of Engineering and Applied Science, George Washington University, Washington D.C., March 1991.

${ }^{43}$ ModelCenter Ver. 6.0, Design Integration Software, Phoenix Integration, Inc., Blacksburg, VA, 2004.

${ }^{44} \mathrm{Li}$, W., Krist, S., and Campbell, R., "Transonic Airfoil Shape Optimization in Preliminary Design Environment," Journal of Aircraft, Vol. 43, No. 3, 2006, pp. 639-651.

${ }^{45}$ Plotkin, K., "Review of Sonic Boom Theory," AIAA-89-1105, AIAA 12th Aeroacoustics Conference, San Antonio, TX, April 1989.

${ }^{46}$ Carlson, H., Chu, J., Ozoroski, L., and McCullers, A., "Guide to AERO2S and WINGDES Computer Codes for Prediction and Minimization of Drag Due to Lift," NASA TP 3637, Nov 1997.

${ }^{47}$ McCullers, A., "Flight Optimization System," NASA Langley Research Center, Hampton, VA, April 2001.

${ }^{48}$ Mangasarian, O., "Normal Solutions of Linear Programs," Mathematical Programming Study, Vol. 22, 1984, pp. 206-216. 


\section{Appendix: Optimization Iterations in BOSS}

1. Range of $\lambda_{s}$. Each constraint in equations (2) and (3) can be expressed as $-\delta \leq \sum_{j=1}^{n-1} \kappa_{j} \Delta r_{j} \leq \delta$, where $\kappa_{j}$ are coefficients for the linear combination. Let $\lambda_{\min }$ be the reciprocal of the maximum value of $\sum_{j=1}^{n-1}\left|\kappa_{j}\right|$ and $\lambda_{\max }=0.2 l^{3}$. Then, the constraints in equations (2) and (3) are automatically satisfied if equation (6) holds for $\lambda_{s}=\lambda_{\min }$. On the other hand, the constraints in equation (6) are automatically satisfied for $\lambda_{s}=\lambda_{\max }$ if equations (2) and (3) hold.

2. Values of $\lambda_{s}$ for $s=0$ or 10. Set $\lambda_{s}=\lambda_{\min }$ (i.e., no smoothness constraint is used) if $s=0$, and set $\lambda_{s}=\lambda_{\min }$ (i.e., the feasible solutions are completely determined by the smoothness constraints) if $s=10$.

3. Estimation of $\lambda_{s}$ for $0<s<10$. Let $\lambda_{s}=1$. Find $\delta>0$ such that the minimum value of $L\left(\mathbf{r}_{0}, \Delta \mathbf{r}\right)$ is $-\rho \hat{G}$ under the constraints in equations (2), (3), and (6). Then, fix $\delta$ and find $\lambda_{s}>0$ such that the ratio of the number of active constraints in equations (2) and (3) to the total number of active constraints is approximately $s / 10$.

4. Initial Reduction. Assume that a total reduction of $\rho \hat{G}$ could be achieved in 100 iterations. Set $\rho_{0}=\rho \hat{G} / 100$ and $k=0$.

5. Iteration for Trusted Reduction. Find $\delta>0$ such that the minimum value of $L\left(\mathbf{r}_{k}, \Delta \mathbf{r}\right)$ is $G\left(\mathbf{r}_{k}\right)-$ $\rho_{k}$ under the constraints in equations (2), (3), and (6). Let $\Delta \mathbf{r}_{k}$ be the solution of the following strictly convex quadratic programming problem:

$$
\min _{\Delta \mathbf{r}} L\left(\mathbf{r}_{k}, \Delta \mathbf{r}\right)+10^{-9} \sum_{i=1}^{n-1} \Delta r\left(x_{i}\right)^{2}
$$

subject to the constraints in equations (2), (3), and (6).

6. Update the Solution. Set $\mathbf{r}_{k+1}=\mathbf{r}_{k}+\Delta \mathbf{r}_{k}$.

7. Check for Termination. If $G\left(\mathbf{r}_{k+1}\right) \leq G\left(\mathbf{r}_{0}\right)-\rho \hat{G}$, then output $\mathbf{r}_{k+1}$ as the solution and terminate the iteration.

8. Reject the Update if Necessary. If $G\left(\mathbf{r}_{k}\right)-G\left(\mathbf{r}_{k+1}\right) \leq 0$, then reset $\mathbf{r}_{k+1}=\mathbf{r}_{k}$.

9. Adjust Reduction Rate if Necessary. If $G\left(\mathbf{r}_{k}\right)-G\left(\mathbf{r}_{k+1}\right)>0.85 \rho_{k}$, then $\rho_{k+1}=2 \rho_{k}$; if $G\left(\mathbf{r}_{k}\right)-$ $G\left(\mathbf{r}_{k+1}\right)<0.35 \rho_{k}$, then $\rho_{k+1}=\rho_{k} / 2$; otherwise, $\rho_{k+1}=\rho_{k}$. If $k<200$, then go back to step 5; otherwise, output $\mathbf{r}_{k+1}$ as the solution and terminate the iteration.

Note that iteration control parameters, such as $\rho_{0}$, the updating formulas for $\rho_{k}$, and the maximum number (200) of iterations, in BOSS are set heuristically based on some preliminary numerical tests. One can use other equally plausible choices. The maximum number of iterations is chosen to achieve a maximum waiting time of 3 min for each BOSS run.

For an inequality constraint $C_{\min } \leq C(\Delta \mathbf{r}) \leq C_{\max }$, the constraint is active for an optimal solution $\Delta \mathbf{r}$ if either $C(\Delta \mathbf{r})=C_{\min }$ or $C(\Delta \mathbf{r})=C_{\max }$. For example, $-\lambda_{s} \delta \leq \Delta \mathbf{r}\left(x_{i}\right) \leq \lambda_{s} \delta$ is called an active constraint if either $\Delta r\left(x_{i}\right)=-\lambda_{s} \delta$ or $\Delta r\left(x_{i}\right)=\lambda_{s} \delta$. The scale $\lambda_{s}$ is a heuristic rule to control the smoothness of shape modifications $\Delta \mathbf{r}$. The number of active constraints in equation (6) implicitly determines how much more influence equation (6) has on the optimal solution than equations (2) and (3). By changing the value of $\lambda_{s}$, we implicitly control the potential influence of equation (6) has on the optimal solution (i.e., the roughness of the shape modification).

Equation (8) is based on Mangasarian's theory on the least norm solution of a linear program. ${ }^{48}$ In theory, one should replace $10^{-9}$ by a sufficiently small positive number to guarantee that the solution of equation (11) is actually a minimizer of $L\left(\mathbf{r}_{k}, \Delta \mathbf{r}\right)$ under the same set of constraints. But $10^{-9}$ works very well in our numerical experiments. In the case without smoothness constraints, Equation (11) forces the optimal solution to stay as close to $\mathbf{r}_{k}$ as possible while achieving the same reduction of the linear approximation $L\left(\mathbf{r}_{k}, \Delta \mathbf{r}\right)$ of the objective function $G$.

Steps 3 and 5 involve solutions of several linear programming problems of less than 200 variables, each of which can be solved in a fraction of seconds. For any given $\delta$, let $L_{k}^{*}(\delta)$ be the minimum value of $L\left(\mathbf{r}_{k}, \Delta \mathbf{r}\right)$ 
under the constraints in equations $(2),(3)$, and $(6)$. Then, $L_{k}^{*}(\delta)$ is a decreasing function of $\delta$ with $L_{k}^{*}(0)=0$. For any given negative target value $L_{\text {target }}<0$, exactly one value of $\delta>0$ exists such that $L_{k}^{*}(\delta)=L_{\text {target }}$. This equation can be efficiently solved by many known methods because of the monotonicity of $L_{k}^{*}(\delta)$. We use a standard root-finding method that uses linear rational function predictions.

\section{The Root-Finding Method Using Linear Rational Function Predictions:}

1. Initialization. Set $\delta_{0}=0$ and $\delta_{1}=1$. Compute $L_{k}^{*}(1)$. Set $\delta_{2}=L_{\text {target }} / L_{k}^{*}(1)$. Compute $L_{k}^{*}\left(\delta_{2}\right)$.

2. Linear Rational Function Interpolation. Let $\eta(t)=\frac{t+c_{0}}{c_{1} t+c_{2}}$ be the interpolant of three data points $\left(\delta_{0}, L_{k}^{*}\left(\delta_{0}\right)\right),\left(\delta_{1}, L_{k}^{*}\left(\delta_{1}\right)\right)$, and $\left(\delta_{2}, L_{k}^{*}\left(\delta_{2}\right)\right)$. That is, $\eta\left(\delta_{i}\right)=L_{k}^{*}\left(\delta_{i}\right)$ for $0 \leq i \leq 2$.

3. Linear Rational Function Prediction. Find $\delta>0$ such that $\eta(\delta)=L_{\text {target }}$ and compute $L_{k}^{*}(\delta)$.

4. Check for Termination. If $\left|L_{k}^{*}(\delta)-L_{\text {target }}\right| /\left|L_{\text {target }}\right|<0.00001$ or a maximum number (40) of iterations is reached, then output $\delta$ as the solution.

5. Update for Next Iteration. Let $j$ be the index such that $\delta_{j}$ is the farthest away from $\delta$ among $\delta_{0}, \delta_{1}$, and $\delta_{2}$. Replace $\delta_{j}$ by $\delta$. Go back to step 2 .

The above root-finding algorithm usually terminates after solving 3-15 linear programming problems because the linear rational function provides an excellent approximation of the nonlinear behavior of $L_{k}^{*}(\delta)$. This algorithm is used in Step 5 of the optimization iterations for BOSS so that the total cost of each iteration in BOSS is approximately that of solving 4-16 linear or quadratic programming problems. For $n=120$, each iteration in BOSS usually takes less than $1 \mathrm{sec}$ when executed on a SGI computer server.

Replacing $\delta$ and $L_{k}^{*}(\delta)$ with $\lambda_{s}$ and the ratio of the number of active constraints in equations (2) and (3) to the total number of active constraints, respectively, we can apply the above root-finding method to find $\lambda_{s}$ in step 2 of Optimization Iterations in BOSS. In this case, we allow the maximum number of iterations to be 80 instead because the value of $\lambda_{s}$ is only estimated once. The average waiting time for BOSS is usually less than 30 sec when $n=120$. 